\title{
SPATIALLY-DISTRIBUTED COVERAGE OPTIMIZATION AND CONTROL WITH LIMITED-RANGE INTERACTIONS
}

\author{
Jorge Cortés $^{1}$, Sonia Martínez ${ }^{2}$ And Francesco Bullo ${ }^{2}$
}

\begin{abstract}
This paper presents coordination algorithms for groups of mobile agents performing deployment and coverage tasks. As an important modeling constraint, we assume that each mobile agent has a limited sensing or communication radius. Based on the geometry of Voronoi partitions and proximity graphs, we analyze a class of aggregate objective functions and propose coverage algorithms in continuous and discrete time. These algorithms have convergence guarantees and are spatially distributed with respect to appropriate proximity graphs. Numerical simulations illustrate the results.
\end{abstract}

Mathematics Subject Classification. 37N35, 49J52, 68W15, 93D20.

Received January 22, 2004. Revised November 18, 2004.

\section{INTRODUCTION}

The current technological development of relatively inexpensive communication, computation, and sensing devices has lead to an intense research activity devoted to the distributed control and coordination of networked systems. In robotic settings, the study of large groups of autonomous vehicles is nowadays a timely concern. The potential advantages of networked robotic systems are their versatility and robustness in the realization of multiple tasks such as manipulation in hazardous environments, pollution detection, estimation and mapbuilding of partially known or unknown environments.

A fundamental problem in the distributed coordination of mobile robots is that of providing stable and decentralized control laws that are scalable with the number of agents in the network. Indeed, since the initial works from the robotics and ecology communities on similar problems on swarms and flocking [1, 25, 28], there have been various efforts to provide rigorous procedures with convergence guarantees using a combination of potential energy shaping methods, gyroscopic forces, and graph theory (see [16,22,26,27] and references therein). In our previous work $[7,8]$, we studied distributed algorithms for deployment and optimal coverage problems using tools from computational geometry, nonsmooth analysis and geometric optimization. The great interest in coordination problems can be easily detected in the proceedings of the 42nd IEEE Conference on Decision and Control held in 2003, the 4th International Conference on Cooperative Control and Optimization held in 2003, or the 6th International Conference on Distributed Autonomous Robotic Systems held in 2002.

\footnotetext{
Keywords and phrases. Distributed dynamical systems, coordination and cooperative control, geometric optimization, nonsmooth analysis, Voronoi partitions.

${ }^{1}$ Department of Applied Mathematics and Statistics, Baskin School of Engineering, University of California, Santa Cruz, 1156 High Street, Santa Cruz, California, 95064, USA; jcortes@ucsc.edu

2 Department of Mechanical and Environmental Engineering, University of California, Santa Barbara, Engineering Building II, Santa Barbara, California, 93106, USA; smartine@engr.ucsb.edu; bullo@engr.ucsb.edu
} 
In devising useful coordination algorithms it is important to progressively account for the various restrictions that real-world systems impose. Building on our previous work [7,8], this paper develops spatially-distributed algorithms for coverage control amenable to implementation on (more) realistic models of networks; we do this by considering the following new aspects. Firstly, we enforce the communication or sensing capacity of an agent to be restricted to a bounded region, typically much smaller than the region where the entire network is confined. In other words, we assume that the agents will have limited-range communication and/or sensing capabilities: we refer to these information exchanges between agents as "limited-range interactions". Secondly, we provide gradient ascent control laws in both continuous and discrete-time settings, and we prove that the induced dynamical systems are convergent. Discrete-time feedback algorithms are indeed the ones truly amenable to implementation in a group of agents exchanging information over a communication network. To deal with these problems, we use a seemingly unrelated combination of tools from graph theory [9], locational optimization [10,23], and systems theory [15].

The contributions of the paper are the following:

(i) Based on the notion of proximity graph [17], we provide a formal notion of spatially-distributed vector fields and maps; we unveil several important properties of the limited-range Delaunay graph, previously introduced in [12], and we show, in a formal way, that it can be computed in a spatially-distributed fashion.

(ii) We analyze the smoothness properties of an important class of objective functions, called multi-center functions, common in locational optimization, quantization theory, and geometric optimization. Our analysis supersedes the results in $[2,10,11,14,23,24]$. One important objective of the analysis is to determine the extent in which certain multi-center functions are spatially distributed and with respect to which proximity graphs.

(iii) We consider the problem of steering the location of a group of robots to local maxima of the objective function. To achieve this objective in continuous and discrete-time, we design novel spatially-distributed control laws for groups of robots. We formally analyze their performance and illustrate their behavior in simulations.

(iv) To perform the smoothness analysis in (ii) and the stability analysis in (iii), we prove useful extensions of the Conservation-of-Mass Law from fluid dynamics and of the discrete-time LaSalle Invariance Principle, respectively. These extensions are, to the best of our knowledge, not present in classical texts on the subject.

It is worth remarking that one fundamental scientific problem in the study of coordination algorithms is scalability with respect to communication complexity. In other words, it is important to design algorithms with communication requirements that scale nicely (e.g., linearly) with the number of agents in the network. However, it is impossible to quantify the communication complexity of any algorithm without introducing a detailed communication model. Adopting a computational geometric approach, this paper classifies the complexity of coordination algorithms in terms of the proximity graphs with respect to which the algorithms are spatially distributed. The underlying assumption is that low complexity proximity graphs (e.g., graphs with a low number of edges) will require limited communication in a realistic implementation.

Throughout the paper we shall consider purposefully only extremely simple models for the dynamics of each individual agent. In particular, we shall assume that the state of each agent is a point in $\mathbb{R}^{2}$ and that the dynamical model of each agent is an integrator (indeed, we shall interchangeably refer to agent as a location or point). This feature is a natural consequence of our focus on network-wide coordination aspects.

The organization of the paper is as follows. In Section 1 we review various preliminary mathematical concepts, and we introduce the notion of proximity graph function and of spatially-distributed map. In Section 2 we study the smoothness of the multi-center function and show in what sense its partial derivative is spatially distributed. In Section 3 we design spatially-distributed coverage algorithms, first in continuous-time and then in discretetime. The numerical outcomes of the algorithms' implementation are reported in Section 4. We discuss possible avenues of future research in Section 5. The appendices present the extensions of the Conservation-of-Mass Law and of the discrete-time LaSalle Invariance Principle. 


\section{Proximity graphs and spatially-Distributed algorithms}

In this section we introduce the notion of proximity graphs and of spatially-distributed algorithms. These notions will later be key in characterizing the distributed character of the proposed coordination algorithms. We start by presenting some preliminary concepts from computational geometry.

\subsection{Preliminaries on graph theory and Voronoi partitions}

Here we gather some basic notions on graph theory and we introduce the notion of Voronoi partition generated by a set of points on the Euclidean space; for comprehensive treatments we refer the reader to [9] and to [4,23], respectively. Given a set $\mathcal{U}$, recall that $2^{\mathcal{U}}$ is the collection of subsets of $\mathcal{U}$. A graph $\mathcal{G}=(\mathcal{U}, \mathcal{E})$ consists of a vertex set $\mathcal{U}$ and an edge set $\mathcal{E} \in 2^{\mathcal{U} \times \mathcal{U}}$. A graph $(\mathcal{U}, \mathcal{E})$ is undirected if $(i, j) \in \mathcal{E}$ implies $(j, i) \in \mathcal{E}$. If $(i, j) \in \mathcal{E}$, then vertex $j$ is a neighbor $\left(\right.$ in $\mathcal{G}$ ) of vertex $i$. Let the map $\mathcal{N}_{\mathcal{G}}: \mathcal{U} \rightarrow 2^{\mathcal{U}}$ associate to the vertex $i$ the set $\mathcal{N}_{\mathcal{G}}(i)$ of its neighbors in $\mathcal{G}$. A graph $\mathcal{G}$ is called complete if any two different vertices in $\mathcal{U}$ are neighbors, i.e., $\mathcal{E}=\mathcal{U} \times \mathcal{U} \backslash \operatorname{diag}(\mathcal{U} \times \mathcal{U})$. This is usually denoted by $K^{n}$. A path connecting vertex $i$ to vertex $j$ is a sequence of vertices $\left\{i_{0}=i, i_{1}, \ldots, i_{k}, i_{k+1}=j\right\}$ with the property that $\left(i_{l}, i_{l+1}\right) \in \mathcal{E}$ for all $l \in\{0, \ldots, k\}$. A graph $\mathcal{G}$ is connected if there exists a path connecting any two vertices of $\mathcal{G}$. Given two graphs $\mathcal{G}_{1}=\left(\mathcal{U}_{1}, \mathcal{E}_{1}\right)$ and $\mathcal{G}_{2}=\left(\mathcal{U}_{2}, \mathcal{E}_{2}\right)$, the intersection graph $\mathcal{G}_{1} \cap \mathcal{G}_{2}$ is the graph $\left(\mathcal{U}_{1} \cap \mathcal{U}_{2}, \mathcal{E}_{1} \cap \mathcal{E}_{2}\right)$, and the union graph $\mathcal{G}_{1} \cup \mathcal{G}_{2}$ is the graph $\left(\mathcal{U}_{1} \cup \mathcal{U}_{2}, \mathcal{E}_{1} \cup \mathcal{E}_{2}\right)$.

A graph $\mathcal{G}_{1}=\left(\mathcal{U}_{1}, \mathcal{E}_{1}\right)$ is a subgraph of a graph $\mathcal{G}_{2}=\left(\mathcal{U}_{2}, \mathcal{E}_{2}\right)$ if $\mathcal{U}_{1} \subseteq \mathcal{U}_{2}$ and $\mathcal{E}_{1} \subseteq \mathcal{E}_{2}$. Alternatively, $\mathcal{G}_{2}$ is said to be a supergraph of $\mathcal{G}_{1}$. Formally, we set $\mathcal{G}_{1} \subseteq \mathcal{G}_{2}$. If $\mathcal{G}_{1} \subseteq \mathcal{G}_{2}$ and $\mathcal{G}_{1}$ contains all the edges $(i, j) \in \mathcal{E}_{2}$ with $i, j \in \mathcal{U}_{1}$, then $\mathcal{G}_{1}$ is called an induced subgraph of $\mathcal{G}_{2}$. A subgraph $\mathcal{G}_{1}$ of $\mathcal{G}_{2}$ is called spanning if $\mathcal{U}_{1}=\mathcal{U}_{2}$. A cycle of $\mathcal{G}$ is a subgraph where every vertex has exactly two neighbors. An acyclic graph is a graph that contains no cycles. A tree is a connected acyclic graph. Given a connected graph $\mathcal{G}$, assign to each edge a positive real number called length or weight. The weight of a subgraph of $\mathcal{G}$ is the sum of the weights of its edges. A minimum spanning tree of $\mathcal{G}$ is a spanning tree with the smallest possible weight. In general, there might exist more than one minimum spanning tree of $\mathcal{G}$, all with the same weight.

Before presenting the notion of Voronoi partition, let us introduce some important notation. Given a vector space $\mathbb{V}$, let $\mathbb{F}(\mathbb{V})$ be the collection of finite subsets of $\mathbb{V}$. Accordingly, $\mathbb{F}\left(\mathbb{R}^{d}\right)$ is the collection of finite point sets in $\mathbb{R}^{d}, d \in \mathbb{N}$; we shall denote an element of $\mathbb{F}\left(\mathbb{R}^{d}\right)$ by $\mathcal{P}=\left\{p_{1}, \ldots, p_{n}\right\} \subset \mathbb{R}^{d}$, where $p_{1}, \ldots, p_{n}$ are distinct points in $\mathbb{R}^{d}$. Let $\mathbb{G}\left(\mathbb{R}^{d}\right)$ be the set of undirected graphs whose vertex set is an element of $\mathbb{F}\left(\mathbb{R}^{d}\right)$.

A covering of $\mathbb{R}^{d}$ is a collection of subsets of $\mathbb{R}^{d}$ whose union is $\mathbb{R}^{d}$; a partition of $\mathbb{R}^{d}$ is a covering whose subsets have disjoint interiors. Let $\mathcal{P} \in \mathbb{F}\left(\mathbb{R}^{d}\right)$ be a set of $n$ distinct points $\left\{p_{1}, \ldots, p_{n}\right\}$ in $\mathbb{R}^{d}$. The Voronoi partition of $\mathbb{R}^{d}$ generated by $\mathcal{P}$ with respect to the Euclidean norm is the collection of $\operatorname{sets}\left\{V_{i}(\mathcal{P})\right\}_{i \in\{1, \ldots, n\}}$ defined by

$$
V_{i}(\mathcal{P})=\left\{q \in \mathbb{R}^{d} \mid\left\|q-p_{i}\right\| \leq\left\|q-p_{j}\right\|, \text { for all } p_{j} \in \mathcal{P}\right\} .
$$

Here, $\|\cdot\|$ denotes the standard Euclidean norm. It is customary and convenient to refer to $V_{i}(\mathcal{P})$ as $V_{i}$. The boundary of each set $V_{i}$ is the union of a finite number of segments and rays.

\subsection{Proximity graphs}

Next, we shall present some relevant concepts on proximity graph functions, that is, on graphs whose vertex set is (in 1-1 correspondence with) a set of distinct points on the Euclidean space and whose edge set is a function of the relative locations of the point set. This notion is an extension of the notion of proximity graph as explained in the survey article [17]; see also [20] and the literature on topology control in wireless networks for related references.

A proximity graph function $\mathcal{G}: \mathbb{F}\left(\mathbb{R}^{d}\right) \rightarrow \mathbb{G}\left(\mathbb{R}^{d}\right)$ associates to a point set $\mathcal{P} \in \mathbb{F}\left(\mathbb{R}^{d}\right)$ an undirected graph with vertex set $\mathcal{P}$ and edge set $\mathcal{E}_{\mathcal{G}}(\mathcal{P})$, where $\mathcal{E}_{\mathcal{G}}: \mathbb{F}\left(\mathbb{R}^{d}\right) \rightarrow \mathbb{F}\left(\mathbb{R}^{d} \times \mathbb{R}^{d}\right)$ has the property that $\mathcal{E}_{\mathcal{G}}(\mathcal{P}) \subseteq$ $\mathcal{P} \times \mathcal{P} \backslash \operatorname{diag}(\mathcal{P} \times \mathcal{P})$ for any $\mathcal{P}$. Here, $\operatorname{diag}(\mathcal{P} \times \mathcal{P})=\{(p, p) \in \mathcal{P} \times \mathcal{P} \mid p \in \mathcal{P}\}$. In other words, the edge set of 
a proximity graph depends on the location of its vertices. We will refer to the map $\mathcal{E}_{\mathcal{G}}$ as the proximity edge function corresponding to the proximity graph function $\mathcal{G}$.

To each proximity graph function $\mathcal{G}$, one can associate the set of neighbors map $\mathcal{N}_{\mathcal{G}}: \mathbb{R}^{d} \times \mathbb{F}\left(\mathbb{R}^{d}\right) \rightarrow \mathbb{F}\left(\mathbb{R}^{d}\right)$, defined by

$$
\mathcal{N}_{\mathcal{G}}(p, \mathcal{P})=\left\{q \in \mathcal{P} \mid(p, q) \in \mathcal{E}_{\mathcal{G}}(\mathcal{P} \cup\{p\})\right\}
$$

Typically, $p$ is a point in $\mathcal{P}$, but the definition is well-posed for any $p \in \mathbb{R}^{d}$. Given $p \in \mathbb{R}^{d}$, it is convenient to define the map $\mathcal{N}_{\mathcal{G}, p}: \mathbb{F}\left(\mathbb{R}^{d}\right) \rightarrow \mathbb{F}\left(\mathbb{R}^{d}\right)$ by $\mathcal{N}_{\mathcal{G}, p}(\mathcal{P})=\mathcal{N}_{\mathcal{G}}(p, \mathcal{P})$. Given two proximity graph functions $\mathcal{G}_{1}$ and $\mathcal{G}_{2}$, we say that $\mathcal{G}_{1}$ is spatially distributed over $\mathcal{G}_{2}$ if, for all $p \in \mathcal{P}$,

$$
\mathcal{N}_{\mathcal{G}_{1}, p}(\mathcal{P})=\mathcal{N}_{\mathcal{G}_{1}, p}\left(\mathcal{N}_{\mathcal{G}_{2}, p}(\mathcal{P})\right)
$$

It is straightforward to deduce that if $\mathcal{G}_{1}$ is spatially distributed over $\mathcal{G}_{2}$, then $\mathcal{G}_{1}$ is a subgraph of $\mathcal{G}_{2}$, that is, $\mathcal{G}_{1}(\mathcal{P}) \subset \mathcal{G}_{2}(\mathcal{P})$ for all $\mathcal{P} \in \mathbb{F}\left(\mathbb{R}^{d}\right)$. The converse is in general not true, as we point out later.

Throughout the rest of the paper, we will focus our attention on the case $d=2$, although most of the discussion can be carried out in arbitrary dimensions. Let $\mathbb{R}_{+}=(0,+\infty)$ and $\overline{\mathbb{R}}_{+}=[0,+\infty)$. For $p \in \mathbb{R}^{2}$ and $r \in \overline{\mathbb{R}}_{+}$, let $\bar{B}(p, r)=\left\{q \in \mathbb{R}^{2} \mid\|q-p\| \leq r\right\}$ and $B(p, r)=\left\{q \in \mathbb{R}^{2} \mid\|q-p\|<r\right\}$ denote the closed and open ball in $\mathbb{R}^{2}$ centered at $p$ of radius $r$, respectively. Now, for $r \in \overline{\mathbb{R}}_{+}$, consider the following proximity graph functions:

(i) the Delaunay graph $\mathcal{P} \mapsto \mathcal{G}_{\mathrm{D}}(\mathcal{P})=\left(\mathcal{P}, \mathcal{E}_{\mathcal{G}_{\mathrm{D}}}(\mathcal{P})\right)$ has edge set

$$
\mathcal{E}_{\mathcal{G}_{\mathrm{D}}}(\mathcal{P})=\left\{\left(p_{i}, p_{j}\right) \in \mathcal{P} \times \mathcal{P} \backslash \operatorname{diag}(\mathcal{P} \times \mathcal{P}) \mid V_{i}(\mathcal{P}) \cap V_{j}(\mathcal{P}) \neq \emptyset\right\}
$$

(ii) the $r$-disk graph $\mathcal{P} \mapsto \mathcal{G}_{\text {disk }}(\mathcal{P}, r)=\left(\mathcal{P}, \mathcal{E}_{\mathcal{G}_{\text {disk }}}(\mathcal{P}, r)\right)$ has edge set

$$
\mathcal{E}_{\mathcal{G}_{\text {disk }}}(\mathcal{P}, r)=\left\{\left(p_{i}, p_{j}\right) \in \mathcal{P} \times \mathcal{P} \backslash \operatorname{diag}(\mathcal{P} \times \mathcal{P}) \mid\left\|p_{i}-p_{j}\right\| \leq r\right\}
$$

(iii) the $r$-Delaunay graph $\mathcal{P} \mapsto \mathcal{G}_{\text {disk } \cap \mathrm{D}}(\mathcal{P}, r)$ is the intersection of $\mathcal{G}_{\text {disk }}(\mathcal{P}, r)$ and $\mathcal{G}_{\mathrm{D}}(\mathcal{P})$;

(iv) the $r$-limited Delaunay (or, limited-range Delaunay) graph $\mathcal{P} \mapsto \mathcal{G}_{\mathrm{LD}}(\mathcal{P}, r)=\left(\mathcal{P}, \mathcal{E}_{\mathcal{G}_{\mathrm{LD}}}(\mathcal{P}, r)\right)$ consists of the edges $\left(p_{i}, p_{j}\right) \in \mathcal{P} \times \mathcal{P} \backslash \operatorname{diag}(\mathcal{P} \times \mathcal{P})$ with the property that

$$
\Delta_{i j}(\mathcal{P}, r) \triangleq\left(V_{i}(\mathcal{P}) \cap \bar{B}\left(p_{i}, \frac{r}{2}\right)\right) \cap\left(V_{j}(\mathcal{P}) \cap \bar{B}\left(p_{j}, \frac{r}{2}\right)\right) \neq \emptyset ;
$$

(v) the Gabriel graph, $\mathcal{P} \mapsto \mathcal{G}_{\mathrm{G}}(\mathcal{P})=\left(\mathcal{P}, \mathcal{E}_{\mathcal{G}_{\mathrm{G}}}(\mathcal{P})\right)$ consists of the edges $\left(p_{i}, p_{j}\right) \in \mathcal{P} \times \mathcal{P} \backslash \operatorname{diag}(\mathcal{P} \times \mathcal{P})$ with the property that

$$
p_{k} \notin B\left(\frac{p_{i}+p_{j}}{2}, \frac{\left\|p_{i}-p_{j}\right\|}{2}\right), \quad \text { for all } k \in\{1, \ldots, n\} \backslash\{i, j\}
$$

(vi) the $r$-Gabriel graph $\mathcal{P} \mapsto \mathcal{G}_{\text {disknG }}(\mathcal{P}, r)$ is the intersection of $\mathcal{G}_{\text {disk }}(\mathcal{P}, r)$ and $\mathcal{G}_{\mathrm{G}}(\mathcal{P})$;

(vii) an Euclidean Minimum Spanning Tree, $\mathcal{P} \mapsto \mathcal{G}_{\mathrm{EMST}}(\mathcal{P})=\left(\mathcal{P}, \mathcal{E}_{\mathcal{G}_{\mathrm{EMST}}}(\mathcal{P})\right.$ ) is defined as a minimum spanning tree of the complete graph $\left(\mathcal{P}, \mathcal{P} \times \mathcal{P} \backslash \operatorname{diag}(\mathcal{P} \times \mathcal{P})\right.$, whose edge $\left(p_{i}, p_{j}\right)$ has weight $\left\|p_{i}-p_{j}\right\|$, for $(i, j) \in\{1, \ldots, n\}$.

Figure 1 presents an example of these proximity graphs for a random configuration of points. In general, one can prove that $\mathcal{G}_{\mathrm{EMST}}(\mathcal{P}) \subseteq \mathcal{G}_{\mathrm{G}}(\mathcal{P}) \subseteq \mathcal{G}_{\mathrm{D}}(\mathcal{P})$ (see for instance [17]). The $r$-Delaunay graph has been studied in $[13,20]$ and the $r$-limited Delaunay graph has been proposed in [12] to develop approximations of geometric objects in the Euclidean space. In the next proposition, we study some basic useful properties of these graphs.

Proposition 1.1. Let $\mathcal{P}=\left\{p_{1}, \ldots, p_{n}\right\} \in \mathbb{F}\left(\mathbb{R}^{2}\right)$, and let $r \in \overline{\mathbb{R}}_{+}$. The following statements hold

(i) $\mathcal{G}_{\text {disk } \cap \mathrm{G}}(\mathcal{P}, r) \subseteq \mathcal{G}_{\mathrm{LD}}(\mathcal{P}, r) \subseteq \mathcal{G}_{\text {disk } \cap \mathrm{D}}(\mathcal{P}, r)$; 


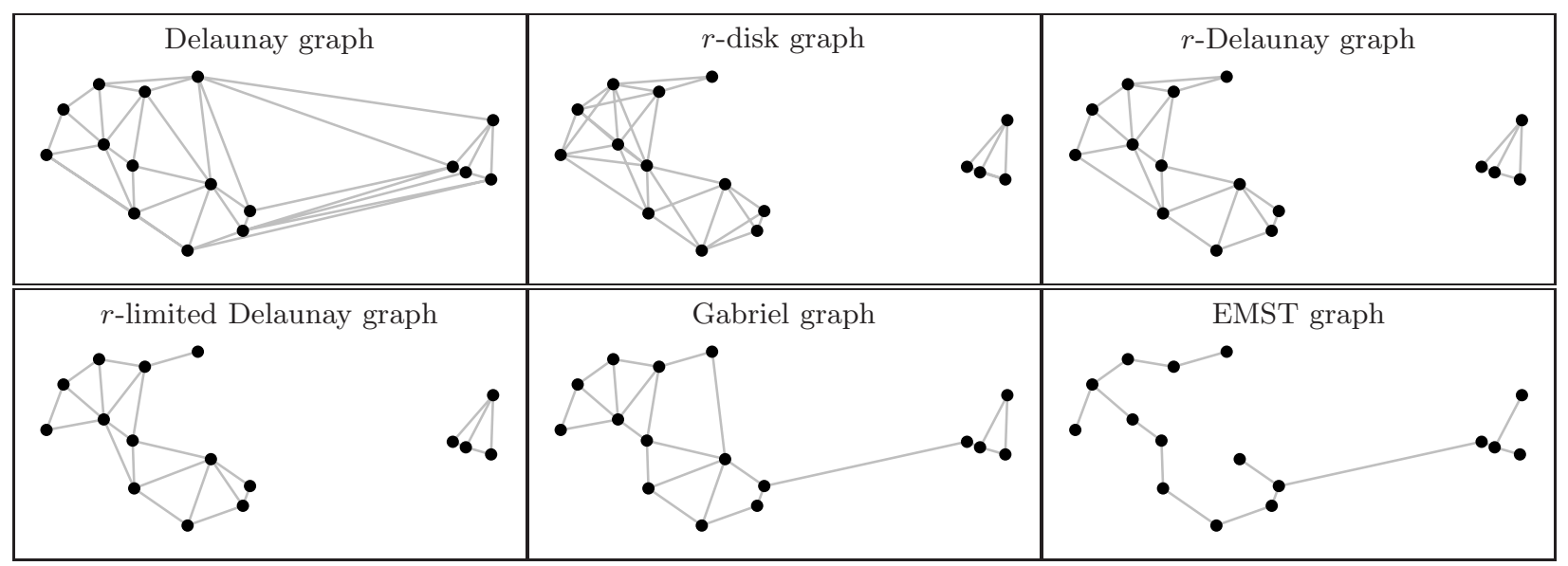

Figure 1. From left to right, and from up to down, Delaunay, $r$-disk, $r$-Delaunay, $r$-limited Delaunay, Gabriel and Euclidean Minimum Spanning Tree graphs for a configuration of 16 generators with coordinates contained in the rectangle $[0,1.9] \times[0,0.75]$. The parameter $r$ is taken equal to 0.45 .

(ii) $\mathcal{G}_{\text {disk }}(\mathcal{P}, r)$ is connected if and only if $\mathcal{G}_{\mathrm{LD}}(\mathcal{P}, r)$ is connected;

(iii) $\# \mathcal{E}_{\mathcal{G}_{\mathrm{LD}}}(\mathcal{P}, r) \leq 3 n-6$ and $\# \mathcal{E}_{\mathcal{G}_{\mathrm{disk}}}(\mathcal{P}, r) \leq \frac{n(n-1)}{2}$. If $\mathcal{G}_{\mathrm{disk}}(\mathcal{P}, r)$ is connected, then $\# \mathcal{E}_{\mathcal{G}_{\mathrm{LD}}}(\mathcal{P}, r) \geq n-1$.

Proof. We first prove the inclusion $\mathcal{G}_{\text {disknG }}(\mathcal{P}, r) \subseteq \mathcal{G}_{\mathrm{LD}}(\mathcal{P}, r)$. Let $\left(p_{i}, p_{j}\right) \in \mathcal{E}_{\mathcal{G}_{\text {disk } \cap \mathrm{G}}}(\mathcal{P}, r)$. From the definition of the Gabriel graph, we deduce that $\left\|\frac{p_{i}+p_{j}}{2}-p_{i}\right\|=\left\|\frac{p_{i}+p_{j}}{2}-p_{j}\right\| \leq\left\|\frac{p_{i}+p_{j}}{2}-p_{k}\right\|$, for all $k \in\{1, \ldots, n\} \backslash\{i, j\}$, and therefore, $\frac{p_{i}+p_{j}}{2} \in V_{i} \cap V_{j}$. Since $\left(p_{i}, p_{j}\right) \in \mathcal{E}_{\mathcal{G}_{\text {disk }}}(\mathcal{P}, r)$, we deduce that $\frac{p_{i}+p_{j}}{2} \in \bar{B}\left(p_{i}, \frac{r}{2}\right) \cap \bar{B}\left(p_{j}, \frac{r}{2}\right)$, and hence equation (1) holds, i.e., $\left(p_{i}, p_{j}\right) \in \mathcal{E}_{\mathcal{G}_{\mathrm{LD}}}(\mathcal{P}, r)$. The second inclusion in (i) is straightforward: if $\left(p_{i}, p_{j}\right) \in \mathcal{E}_{\mathcal{G}_{\mathrm{LD}}}(\mathcal{P}, r)$, then equation $(1)$ implies that $V_{i}(\mathcal{P}) \cap V_{j}(\mathcal{P}) \neq \emptyset$, i.e., $\left(p_{i}, p_{j}\right) \in \mathcal{E}_{\mathcal{G}_{\mathrm{D}}}(\mathcal{P})$. Since clearly $\left(p_{i}, p_{j}\right) \in \mathcal{E}_{\mathcal{G}_{\text {disk }}}(\mathcal{P}, r)$, we conclude (i). The statement (ii) is a consequence of the following more general fact: the $r$-disk graph $\mathcal{G}_{\text {disk }}(\mathcal{P}, r)$ is connected if and only if $\mathcal{G}_{\mathrm{EMST}}(\mathcal{P}) \subseteq \mathcal{G}_{\text {disk }}(\mathcal{P}, r)$. The proof of this fact is as follows. If $\mathcal{G}_{\mathrm{EMST}}(\mathcal{P}) \subseteq \mathcal{G}_{\text {disk }}(\mathcal{P}, r)$, then clearly $\mathcal{G}_{\text {disk }}(\mathcal{P}, r)$ is connected. To prove the other implication, assume that $\mathcal{G}_{\text {disk }}(\mathcal{P}, r)$ is connected. We reason by contradiction. Let $\mathcal{G}_{\text {EMST }}(\mathcal{P}) \nsubseteq \mathcal{G}_{\text {disk }}(\mathcal{P}, r)$, i.e., there exists $p_{i}$ and $p_{j}$ with $\left(p_{i}, p_{j}\right) \in \mathcal{E}_{\mathcal{G}_{\mathrm{EMST}}}(\mathcal{P})$ and $\left\|p_{i}-p_{j}\right\|>r$. If we remove this edge from $\mathcal{E}_{\mathcal{G}_{\mathrm{EMST}}}(\mathcal{P})$, the tree becomes disconnected into two connected components $T_{1}$ and $T_{2}$, with $p_{i} \in T_{1}$ and $p_{j} \in T_{2}$. Now, since by hypothesis the $r$-disk graph $\mathcal{G}_{\text {disk }}(\mathcal{P}, r)$ is connected, there must exist $k, l \in\{1, \ldots, n\}$ such that $p_{k} \in T_{1}, p_{l} \in T_{2}$ and $\left\|p_{k}-p_{l}\right\| \leq r$. If we add the edge $\left(p_{k}, p_{l}\right)$ to the set of edges of $T_{1} \cup T_{2}$, the obtained graph $\mathcal{G}$ is acyclic, connected and contains all the vertices $\mathcal{P}$, i.e., $\mathcal{G}$ is a spanning tree. Moreover, since $\left\|p_{k}-p_{l}\right\| \leq r<\left\|p_{i}-p_{j}\right\|$ and $T_{1}$ and $T_{2}$ are induced subgraphs of $\mathcal{G}_{\mathrm{EMST}}(\mathcal{P})$, we conclude that $\mathcal{G}$ has smaller length than $\mathcal{G}_{\mathrm{EMST}}(\mathcal{P}, r)$, which is a contradiction. As a consequence, we deduce that if $\mathcal{G}_{\text {disk }}(\mathcal{P}, r)$ is connected, then $\mathcal{G}_{\mathrm{EMST}}(\mathcal{P}) \subseteq \mathcal{G}_{\text {disknG }}(\mathcal{P}, r)$. Using (i), we conclude that $\mathcal{G}_{\mathrm{LD}}(\mathcal{P}, r)$ is connected. Finally, the bounds concerning the $r$-limited Delaunay graph in (iii) follow from (i) and (ii) by noting that $\# \mathcal{E}_{\mathcal{G}_{\mathrm{D}}}(\mathcal{P}) \leq 3 n-6$ (see, for instance, [23]) and $\# \mathcal{E}_{\mathcal{G}_{\mathrm{EMST}}}(\mathcal{P})=n-1$. The bound on the $r$-disk graph follows from this graph being a subgraph of the complete graph.

Let us make the following observations concerning Proposition 1.1.

Remarks 1.2. As before, let $\mathcal{P}=\left\{p_{1}, \ldots, p_{n}\right\} \in \mathbb{F}\left(\mathbb{R}^{2}\right)$ and let $r \in \overline{\mathbb{R}}_{+}$.

(i) The $r$-Delaunay graph does not coincide in general with the $r$-limited Delaunay graph. Figure 2 illustrates a point set $\mathcal{P}$ in which $p_{l}$ is a neighbor of $p_{i}$ in $\mathcal{G}_{\text {disknD }}(\mathcal{P}, r)$ but not in $\mathcal{G}_{\mathrm{LD}}(\mathcal{P}, r)$.

(ii) The collection $\left\{V_{i}(\mathcal{P}) \cap \bar{B}\left(p_{i}, \frac{r}{2}\right)\right\}_{i \in\{1, \ldots, n\}}$ is a partition of the set $\cup_{i=1}^{n} \bar{B}\left(p_{i}, \frac{r}{2}\right) \subset \mathbb{R}^{2}$. The boundary of each set $V_{i}(\mathcal{P}) \cap \bar{B}\left(p_{i}, \frac{r}{2}\right), i \in\{1, \ldots, n\}$, is the union of a finite number of segments and arcs; see 


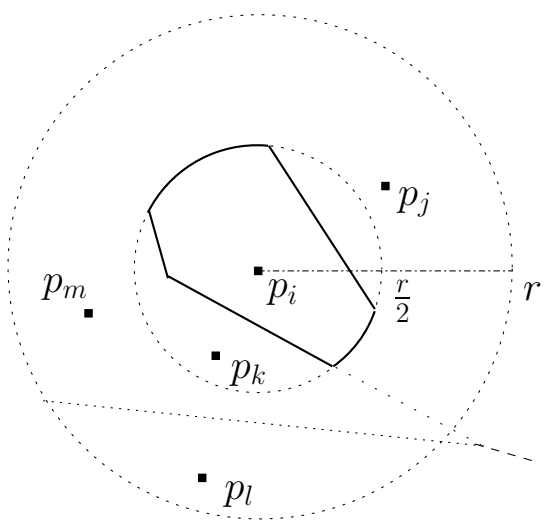

FiguRE 2. Example point set for which the $r$-Delaunay graph strictly contains the $r$-limited Delaunay graph: $p_{l}$ is a neighbor of $p_{i}$ in $\mathcal{G}_{\text {disknD }}(\mathcal{P}, r)$ but not in $\mathcal{G}_{\mathrm{LD}}(\mathcal{P}, r)$.
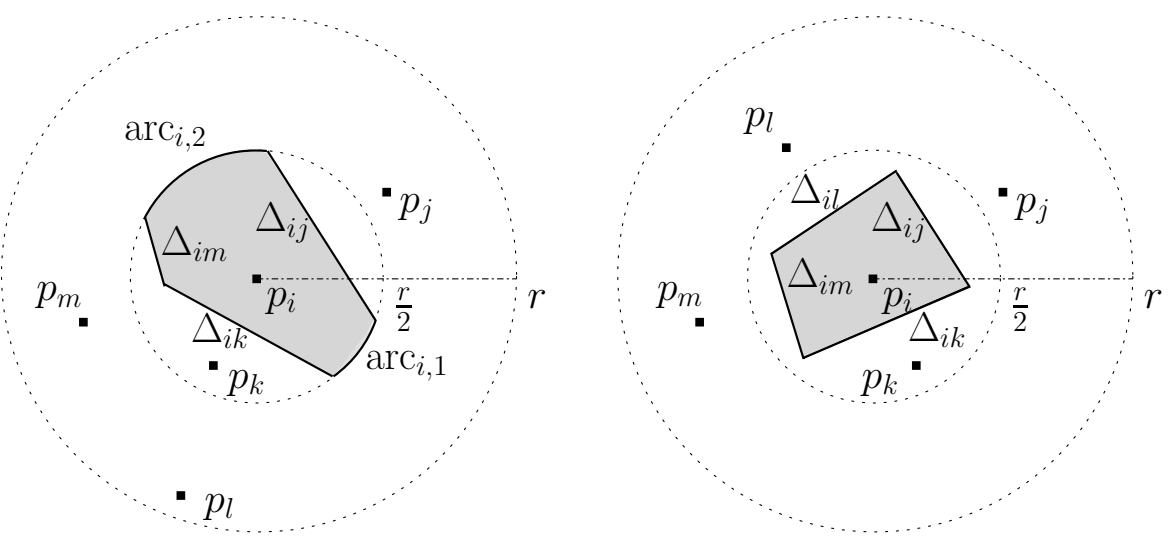

FIgURE 3. The shaded regions are examples of sets $V_{i}(\mathcal{P}) \cap \bar{B}\left(p_{i}, \frac{r}{2}\right)$. In the right figure, the boundary of the set $V_{i}(\mathcal{P}) \cap \bar{B}\left(p_{i}, \frac{r}{2}\right)$ does not contain any arc.

Figure 3. Therefore, at fixed $\mathcal{P}$, there exist $n$ numbers $M_{i}(r) \geq 0, i \in\{1, \ldots, n\}$, of distinct arcs $\operatorname{arc}_{i, 1}(r), \ldots, \operatorname{arc}_{i, M_{i}(r)}(r)$ of spheres of radius $\frac{r}{2}$ in $\partial\left(V_{i}(\mathcal{P}) \cap \bar{B}\left(p_{i}, \frac{r}{2}\right)\right)$ with the property that

$$
\partial\left(V_{i}(\mathcal{P}) \cap \bar{B}\left(p_{i}, \frac{r}{2}\right)\right)=\left(\cup_{j \in \mathcal{N}_{\mathcal{G}_{\mathrm{LD}}(\mathcal{P}, r)}\left(p_{i}\right)} \Delta_{i j}(r)\right) \cup\left(\cup_{l \in\left\{1, \ldots, M_{i}(r)\right\}} \operatorname{arc}_{i, l}(r)\right),
$$

where we recall that $\mathcal{N}_{\mathcal{G}_{\mathrm{LD}}(\mathcal{P}, r)}\left(p_{i}\right)$ denotes the set of neighbors in $\mathcal{G}_{\mathrm{LD}}(\mathcal{P}, r)$ of the vertex $p_{i}$.

(iii) If $\left\|p_{i}-p_{j}\right\|$ is strictly greater than $r$, then the half plane $\left\{q \in \mathbb{R}^{2} \mid\left\|q-p_{i}\right\| \leq\left\|q-p_{j}\right\|\right\}$ contains the ball $\bar{B}\left(p_{i}, \frac{r}{2}\right)$. Accordingly,

$$
\begin{aligned}
\bar{B}\left(p_{i}, \frac{r}{2}\right) \cap V_{i}(\mathcal{P}) & =\bar{B}\left(p_{i}, \frac{r}{2}\right) \cap\left\{q \in \mathbb{R}^{2} \mid\left\|q-p_{i}\right\| \leq\left\|q-p_{j}\right\|, \text { for all } p_{j} \in \mathcal{P}\right\} \\
& =\bar{B}\left(p_{i}, \frac{r}{2}\right) \cap\left\{q \in \mathbb{R}^{2} \mid\left\|q-p_{i}\right\| \leq\left\|q-p_{j}\right\|, \text { for all } p_{j} \in \mathcal{N}_{\mathcal{G}_{\text {disk }}(\mathcal{P}, r)}\left(p_{i}\right)\right\}
\end{aligned}
$$

(iv) For simplicity, it is convenient to refer to the various proximity graphs functions without explicitly writing the argument $\mathcal{P}$.

Finally, we conclude this section with a general note. 
Remark 1.3. The notion of proximity graph is defined for sets of distinct points $\mathcal{P} \in \mathbb{F}\left(\mathbb{R}^{d}\right)$. However, we will often consider tuples of elements of $\mathbb{R}^{d}$ of the form $P=\left(p_{1}, \ldots, p_{n}\right)$, i.e., ordered sets of possibly coincident points. Let $i_{\mathbb{F}}:\left(\mathbb{R}^{d}\right)^{n} \rightarrow \mathbb{F}\left(\mathbb{R}^{d}\right)$ be the natural immersion, i.e., $i_{\mathbb{F}}(P)$ is the point set that contains only the distinct points in $P=\left(p_{1}, \ldots, p_{n}\right)$. In what follows, $\mathcal{P}=i_{\mathbb{F}}(P)$ will always denote the point set associated to $P \in\left(\mathbb{R}^{d}\right)^{n}$. Note that $i_{\mathbb{F}}$ is invariant under permutations of its arguments and that the cardinality of $i_{\mathbb{F}}\left(p_{1}, \ldots, p_{n}\right)$ is in general less than or equal to $n$. More precisely, if $\mathcal{S}$ denotes the set

$$
\mathcal{S}=\left\{\left(p_{1}, \ldots, p_{n}\right) \in\left(\mathbb{R}^{d}\right)^{n} \mid p_{i}=p_{j} \text { for some } i, j \in\{1, \ldots, n\}, i \neq j\right\},
$$

then $\# \mathcal{P}<n$ if $\left(p_{1}, \ldots, p_{n}\right) \in \mathcal{S}$ and $\# \mathcal{P}=n$ if $\left(p_{1}, \ldots, p_{n}\right) \notin \mathcal{S}$. The Voronoi covering $\mathcal{V}\left(p_{1}, \ldots, p_{n}\right)=$ $\left\{V_{i}\left(p_{1}, \ldots, p_{n}\right)\right\}_{i \in\{1, \ldots, n\}}$ generated by the tuple $\left(p_{1}, \ldots, p_{n}\right)$ is defined by assigning to each point $p_{i}$ its corresponding Voronoi cell in the Voronoi partition generated by $\mathcal{P}$. Note that coincident points in the tuple $\left(p_{1}, \ldots, p_{n}\right)$ have the same Voronoi cell.

We can now extend the notion of proximity graphs to this setting. Given a proximity graph function $\mathcal{G}$ with edge set function $\mathcal{E}_{\mathcal{G}}$, we define (with a slight abuse of notation)

$$
\begin{aligned}
& \mathcal{G}=\mathcal{G} \circ i_{\mathbb{F}}:\left(\mathbb{R}^{d}\right)^{n} \rightarrow \mathbb{G}\left(\mathbb{R}^{d}\right), \\
& \mathcal{E}_{\mathcal{G}}=\mathcal{E}_{\mathcal{G}} \circ i_{\mathbb{F}}:\left(\mathbb{R}^{d}\right)^{n} \rightarrow \mathbb{F}\left(\mathbb{R}^{d} \times \mathbb{R}^{d}\right) .
\end{aligned}
$$

Additionally, we define the set of neighbors map $\mathcal{N}_{\mathcal{G}}:\left(\mathbb{R}^{d}\right)^{n} \rightarrow\left(\mathbb{F}\left(\mathbb{R}^{d}\right)\right)^{n}$ as the function whose $j$ th component is

$$
\mathcal{N}_{\mathcal{G}, j}\left(p_{1}, \ldots, p_{n}\right)=\mathcal{N}_{\mathcal{G}}\left(p_{j}, i_{\mathbb{F}}\left(p_{1}, \ldots, p_{n}\right)\right)
$$

Note that coincident points in the tuple $\left(p_{1}, \ldots, p_{n}\right)$ will have the same set of neighbors.

\subsection{Spatially-distributed functions, vector fields, and set-valued maps}

We are now in a position to discuss distributed control laws and algorithms in formal terms. From now on, we shall deal not only with sets of distinct points, but also with tuples of elements of $\mathbb{R}^{2}$. Let $\mathcal{G}$ be a proximity graph function and let $Y$ be a set. A map $f:\left(\mathbb{R}^{2}\right)^{n} \rightarrow Y^{n}$ is spatially distributed over $\mathcal{G}$ if there exist maps $\tilde{f}_{i}: \mathbb{R}^{2} \times \mathbb{F}\left(\mathbb{R}^{2}\right) \rightarrow Y, i \in\{1, \ldots, n\}$, with the property that for all $\left(p_{1}, \ldots, p_{n}\right) \in\left(\mathbb{R}^{2}\right)^{n}$

$$
f_{i}\left(p_{1}, \ldots, p_{n}\right)=\tilde{f}_{i}\left(p_{i}, \mathcal{N}_{\mathcal{G}, i}\left(p_{1}, \ldots, p_{n}\right)\right),
$$

where $f_{i}$ denotes the $i$ th-component of $f$. A vector field $X$ on $\left(\mathbb{R}^{2}\right)^{n}$ is spatially distributed over $\mathcal{G}$ if its associated map $X:\left(\mathbb{R}^{2}\right)^{n} \rightarrow\left(\mathbb{R}^{2}\right)^{n}$, where the canonical identification between the tangent space of $\left(\mathbb{R}^{2}\right)^{n}$ and $\left(\mathbb{R}^{2}\right)^{n}$ itself is understood, is spatially distributed in the above sense. Likewise, a set-valued map $T:\left(\mathbb{R}^{2}\right)^{n} \rightarrow 2^{Y^{n}} \equiv\left(2^{Y}\right)^{n}$ is spatially distributed over $\mathcal{G}$ if there exist maps $\tilde{T}_{i}: \mathbb{R}^{2} \times \mathbb{F}\left(\mathbb{R}^{2}\right) \rightarrow 2^{Y}, i \in\{1, \ldots, n\}$, with the property that for all $\left(p_{1}, \ldots, p_{n}\right) \in\left(\mathbb{R}^{2}\right)^{n}$

$$
T_{i}\left(p_{1}, \ldots, p_{n}\right)=\tilde{T}_{i}\left(p_{i}, \mathcal{N}_{\mathcal{G}, i}\left(p_{1}, \ldots, p_{n}\right)\right)
$$

where $T_{i}$ denotes the $i$ th-component of $T$.

Remark 1.4. In other words, to compute the $i$ th component of a spatially-distributed function, vector field, or set-valued map at $\left(p_{1}, \ldots, p_{n}\right)$, it is only required the knowledge of the vertex $p_{i}$ and the neighboring vertices in the graph $\mathcal{G}\left(\left\{p_{1}, \ldots, p_{n}\right\}\right)$.

Remark 1.5. One can see that the proximity graph function $\mathcal{G}_{1}$ is spatially distributed over the proximity graph function $\mathcal{G}_{2}$ if and only if the set of neighbors map $\mathcal{N}_{\mathcal{G}_{1}}$ is spatially distributed over $\mathcal{G}_{2}$ according to the previous definition.

We are now in a position to establish an important property of the $r$-limited Delaunay graph. 
Lemma 1.6. Let $r \in \overline{\mathbb{R}}_{+}$. Then, the $r$-limited Delaunay graph $\mathcal{G}_{\mathrm{LD}}(r)$ is spatially distributed over $\mathcal{G}_{\text {disk }}(r)$, i.e., the set of neighbors map $\mathcal{N}_{\mathcal{G}_{\mathrm{LD}}(r)}:\left(\mathbb{R}^{2}\right)^{n} \rightarrow\left(\mathbb{F}\left(\mathbb{R}^{2}\right)\right)^{n}$ is spatially distributed over $\mathcal{G}_{\text {disk }}(r)$.

Proof. The result follows from Remark 1.2(iii).

Loosely speaking, this lemma states that the $r$-limited Delaunay graph $\mathcal{G}_{\mathrm{LD}}$ can be computed in a spatially localized way: each agent needs to know only the location of all other agents in a disk of radius $r$. This property is to be contrasted with the centralized computation required to determine the $r$-Delaunay graph $\mathcal{G}_{\text {disk } \cap \mathrm{D}}$. This requirement can be observed in Figure 2: if $p_{j}$ and $p_{l}$ are placed arbitrarily close to the line joining $p_{i}$ and $p_{k}$, then, in order to decide if $p_{l} \in \mathcal{N}_{\mathcal{G}_{\text {disknD }}(\mathcal{P}, r)}\left(p_{i}\right)$, in general it is necessary to know the locations of all the other points in $\left\{p_{1}, \ldots, p_{n}\right\}$. That is, $\mathcal{G}_{\text {disk } \cap \mathrm{D}} \subset \mathcal{G}_{\text {disk }}$ is not spatially distributed over $\mathcal{G}_{\text {disk }}$.

\section{LiMITED-RANGE LOCATIONAL OPTIMIZATION}

Let $Q$ be a simple convex polygon in $\mathbb{R}^{2}$ including its interior. The diameter of $Q$ is defined as $\operatorname{diam}(Q)=$ $\max _{q, p \in Q}\|q-p\|$. For $\delta, \epsilon \in \overline{\mathbb{R}}_{+}, \delta<\epsilon$, let $D(p,[\delta, \epsilon])=\left\{q \in \mathbb{R}^{2} \mid \delta \leq\|q-p\| \leq \epsilon\right\}$ denote the annulus in $\mathbb{R}^{2}$ centered at $p$ of radius $\delta$ and $\epsilon$; it is also convenient to define $D(p,[0, \epsilon])=\bar{B}(p, \epsilon)=\left\{q \in \mathbb{R}^{2} \mid\|q-p\| \leq \epsilon\right\}$ and $D(p,[\delta,+\infty])=\left\{q \in \mathbb{R}^{2} \mid \delta \leq\|q-p\|\right\}$. Let $n_{\bar{B}(p, \epsilon)}(q)$ denote the unit outward normal to $\bar{B}(p, \epsilon)$ at $q \in \partial \bar{B}(p, \epsilon)$. Given a set $S \subset Q$, let $1_{S}$ denote the indicator function defined by $1_{S}(q)=1$ if $q \in S$, and $1_{S}(q)=0$ if $q \notin S$.

Throughout the rest of the paper, given a set of $n$ distinct points $\mathcal{P}=\left\{p_{1}, \ldots, p_{n}\right\} \in \mathbb{F}\left(\mathbb{R}^{2}\right)$, we consider the restriction of the Voronoi partition $\mathcal{V}(\mathcal{P})$ generated by $\mathcal{P}$ to the convex polygon $Q,\left\{V_{i}(\mathcal{P}) \cap Q\right\}_{i \in\{1, \ldots, n\}}$. For ease of exposition, we denote this restriction in the same way as the standard Voronoi partition. Given a tuple $P=\left(p_{1}, \ldots, p_{n}\right) \in Q^{n}$, recall that we let $\mathcal{P}=\left\{p_{1}, \ldots, p_{n}\right\}$ denote the associated point set that only contains the corresponding distinct points.

A density function $\phi: Q \rightarrow \overline{\mathbb{R}}_{+}$is a bounded measurable function on $Q$. Given a set $S \subset Q$, let area $\phi(S)$ denote the area of $S$ measured according to $\phi$, i.e., $\operatorname{area}_{\phi}(S)=\int_{S} \phi(q) \mathrm{d} q$. A performance function $f: \overline{\mathbb{R}}_{+} \rightarrow \mathbb{R}$ is a non-increasing and piecewise differentiable map with finite jump discontinuities at $R_{1}, \ldots, R_{m} \in \mathbb{R}_{+}=$ $(0,+\infty)$, with $R_{1}<\cdots<R_{m}$. For convenience, we set $R_{0}=0$ and $R_{m+1}=+\infty$, and write

$$
f(x)=\sum_{\alpha=1}^{m+1} f_{\alpha}(x) 1_{\left[R_{\alpha-1}, R_{\alpha}\right)}(x)
$$

where $f_{\alpha}:\left[R_{\alpha-1}, R_{\alpha}\right] \rightarrow \mathbb{R}, \alpha \in\{1, \ldots, m+1\}$ are non-increasing continuously differentiable functions such that $f_{\alpha}\left(R_{\alpha}\right)>f_{\alpha+1}\left(R_{\alpha}\right)$ for $\alpha \in\{1, \ldots, m\}$. Given a density function $\phi$ and a performance function $f$, we consider the multi-center function $\mathcal{H}: Q^{n} \rightarrow \mathbb{R}$ defined by

$$
\mathcal{H}(P)=\int_{Q} \max _{i \in\{1, \ldots, n\}} f\left(\left\|q-p_{i}\right\|\right) \phi(q) \mathrm{d} q
$$

Note that $\mathcal{H}$ is an aggregate objective function in the sense that it depends on all the locations $p_{1}, \ldots, p_{n}$. It will be of interest to find local maxima for $\mathcal{H}$. Note that the performance function $f$ can be defined up to a constant $c \in \mathbb{R}$, since

$$
\int_{Q}\left(\max _{i \in\{1, \ldots, n\}}\left(f\left(\left\|q-p_{i}\right\|\right)+c\right)\right) \phi(q) \mathrm{d} q=\mathcal{H}(P)+c \operatorname{area}_{\phi}(Q)
$$

and, therefore, this function and $\mathcal{H}$ have the same local maxima.

Remark 2.1. Maximizing the multi-center function is an optimal resource placement problem; it is interesting to draw an analogy with the optimal placement problem for large numbers of spatially-distributed sensors. 
In this setting, (1) $\mathcal{H}$ provides the expected value of the sensing performance provided by the group of sensors over any point in the environment $Q$, where (2) the function $\phi$ is the distribution density function representing a measure of information or probability that some event take place over $Q$, and (3) $f$ describes the utility or sensing performance of the sensors. Because of noise and loss of resolution, the sensing performance at point $q$ taken from $i$ th sensor at the position $p_{i}$ degrades with the distance $\left\|q-p_{i}\right\|$ between $q$ and $p_{i}$.

Let us provide two equivalent expressions for the function $\mathcal{H}$ over the set $Q^{n} \backslash \mathcal{S}$, for $\mathcal{S}$ defined in equation (3). Using the definition of the Voronoi partition and the fact that $f$ is non-increasing, $\mathcal{H}$ can be rewritten as

$$
\mathcal{H}(P)=\sum_{i=1}^{n} \int_{V_{i}(P)} f\left(\left\|q-p_{i}\right\|\right) \phi(q) \mathrm{d} q, \quad P \in Q^{n} \backslash \mathcal{S} .
$$

Resorting to the expression of $f$ in (4), we can also rewrite $\mathcal{H}$ as

$$
\mathcal{H}(P)=\sum_{i=1}^{n} \sum_{\alpha=1}^{m+1} \int_{V_{i}(P) \cap D\left(p_{i},\left[R_{\alpha-1}, R_{\alpha}\right]\right)} f_{\alpha}\left(\left\|q-p_{i}\right\|\right) \phi(q) \mathrm{d} q, \quad P \in Q^{n} \backslash \mathcal{S} .
$$

We now analyze the smoothness properties of the multi-center function.

Theorem 2.2. Given a density function $\phi$ and a performance function $f$, the multi-center function $\mathcal{H}$ is

(i) globally Lipschitz on $Q^{n}$, and

(ii) continuously differentiable on $Q^{n} \backslash \mathcal{S}$, where for each $i \in\{1, \ldots, n\}$

$$
\begin{aligned}
\frac{\partial \mathcal{H}}{\partial p_{i}}(P)= & \int_{V_{i}(P)} \frac{\partial}{\partial p_{i}} f\left(\left\|q-p_{i}\right\|\right) \phi(q) \mathrm{d} q \\
& +\sum_{\alpha=1}^{m}\left(f_{\alpha}\left(R_{\alpha}\right)-f_{\alpha+1}\left(R_{\alpha}\right)\right)\left(\sum_{k=1}^{M_{i}\left(2 R_{\alpha}\right)} \int_{\operatorname{arc}_{i, k}\left(2 R_{\alpha}\right)} n_{\bar{B}\left(p_{i}, R_{\alpha}\right)}(q) \phi(q) \mathrm{d} q\right),
\end{aligned}
$$

with $\operatorname{arc}_{i, k}\left(2 R_{\alpha}\right), k \in\left\{1, \ldots, M_{i}\left(2 R_{\alpha}\right)\right\}$ the arcs in the boundary of $V_{i}(P) \cap \bar{B}\left(p_{i}, R_{\alpha}\right)$.

Proof. We start by proving fact (i). Because $\max _{i \in\{1, \ldots, n\}}\left\|q-p_{i}\right\| \leq \operatorname{diam}(Q)$ for all $q, p_{1}, \ldots, p_{n}$ in $Q$, we can assume, without loss of generality, that $R_{m+1}=\operatorname{diam}(Q)$. Since the functions $f_{\alpha}, \alpha \in\{1, \ldots, m+1\}$, are continuously differentiable on $\left[R_{\alpha-1}, R_{\alpha}\right]$, they admit a non-increasing $C^{1}$-extension to $\left[0, R_{\alpha}\right]$, that we also denote by $f_{\alpha}$ for simplicity. We then rewrite $\mathcal{H}$ as

$$
\mathcal{H}(P)=\sum_{\alpha=1}^{m+1} \int_{Q} f_{\alpha}(\operatorname{dist}(q, P))\left(1_{\left[0, R_{\alpha}\right)}(\operatorname{dist}(q, P))-1_{\left[0, R_{\alpha-1}\right)}(\operatorname{dist}(q, P)) \phi(q) \mathrm{d} q,\right.
$$

where $\operatorname{dist}(q, P)=\min _{i \in\{1, \ldots, n\}}\left\|q-p_{i}\right\|$, for $P=\left(p_{1}, \ldots p_{n}\right) \in Q^{n}$. Since the finite sum of globally Lipschitz functions is globally Lipschitz, in what follows it suffices to prove that for $R \in\left[0, R_{\alpha}\right]$ and for $\alpha \in\{1, \ldots, m+1\}$, the function

$$
\mathcal{H}_{\alpha, R}(P)=\int_{Q} f_{\alpha}(\operatorname{dist}(q, P)) 1_{[0, R)}(\operatorname{dist}(q, P)) \phi(q) \mathrm{d} q
$$


is globally Lipschitz. To this end, we introduce a useful partition of $Q$. For $S \subset Q$, recall $S^{c}=Q \backslash S$. Given $P=\left(p_{1}, \ldots, p_{n}\right), P^{\prime}=\left(p_{1}^{\prime}, \ldots, p_{n}^{\prime}\right)$, define the following sets

$$
\begin{aligned}
& S_{1}=\left(\cup_{i \in\{1, \ldots, n\}} \bar{B}\left(p_{i}, R\right)\right) \cap\left(\cap_{i \in\{1, \ldots, n\}} \bar{B}\left(p_{i}^{\prime}, R\right)^{c}\right), \\
& S_{2}=\left(\cup_{i \in\{1, \ldots, n\}} \bar{B}\left(p_{i}^{\prime}, R\right)\right) \cap\left(\cap_{i \in\{1, \ldots, n\}} \bar{B}\left(p_{i}, R\right)^{c}\right), \\
& S_{3}=\left(\cap_{i \in\{1, \ldots, n\}} \bar{B}\left(p_{i}, R\right)^{c}\right) \cap\left(\cap_{i \in\{1, \ldots, n\}} \bar{B}\left(p_{i}^{\prime}, R\right)^{c}\right), \\
& S_{4}=\left(\cup_{i \in\{1, \ldots, n\}} \bar{B}\left(p_{i}, R\right)\right) \cap\left(\cup_{i \in\{1, \ldots, n\}} \bar{B}\left(p_{i}^{\prime}, R\right)\right) .
\end{aligned}
$$

Note that $S_{1} \cup S_{2}=\left(S_{3} \cup S_{4}\right)^{c}$ and therefore $Q=S_{1} \cup S_{2} \cup S_{3} \cup S_{4}$. Also, observe that $S_{a} \cap S_{b}=\emptyset$ for any $a$, $b \in\{1,2,3,4\}$ with $a \neq b$. Accordingly, we write

$$
\mathcal{H}_{\alpha, R}(P)-\mathcal{H}_{\alpha, R}\left(P^{\prime}\right)=\sum_{a=1}^{4} \int_{S_{a}}\left(f_{\alpha}(\operatorname{dist}(q, P)) 1_{[0, R)}(\operatorname{dist}(q, P))-f_{\alpha}\left(\operatorname{dist}\left(q, P^{\prime}\right)\right) 1_{[0, R)}\left(\operatorname{dist}\left(q, P^{\prime}\right)\right)\right) \phi(q) \mathrm{d} q .
$$

Now we upper bound each of the integrals in the above sum. For $q \in S_{3}$, we have $1_{[0, R)}(\operatorname{dist}(q, P))=0$ and $1_{[0, R)}\left(\operatorname{dist}\left(q, P^{\prime}\right)\right)=0$, and therefore the integral over $S_{3}$ vanishes. For $q \in S_{4}$, we have $\operatorname{dist}(q, P) \leq R$ and $\operatorname{dist}\left(q, P^{\prime}\right) \leq R$. Thus,

$$
\begin{aligned}
\mid \int_{S_{4}}\left(f_{\alpha}(\operatorname{dist}(q, P))\right. & \left.1_{[0, R)}(\operatorname{dist}(q, P))-f_{\alpha}\left(\operatorname{dist}\left(q, P^{\prime}\right)\right) 1_{[0, R)}\left(\operatorname{dist}\left(q, P^{\prime}\right)\right)\right) \phi(q) \mathrm{d} q \mid \\
& \leq \int_{S_{4}}\left|f_{\alpha}(\operatorname{dist}(q, P))-f_{\alpha}\left(\operatorname{dist}\left(q, P^{\prime}\right)\right)\right| \phi(q) \mathrm{d} q \\
& \leq\left\|\frac{\mathrm{d} f_{\alpha}}{\mathrm{d} x}\right\|_{\left[0, R_{\alpha}\right]} \int_{S_{4}}\left|\operatorname{dist}(q, P)-\operatorname{dist}\left(q, P^{\prime}\right)\right| \phi(q) \mathrm{d} q \\
& \leq\left\|\frac{\mathrm{d} f_{\alpha}}{\mathrm{d} x}\right\|_{\left[0, R_{\alpha}\right]}\left\|P-P^{\prime}\right\| \int_{S_{4}} \phi(q) \mathrm{d} q \leq\left\|\frac{\mathrm{d} f_{\alpha}}{\mathrm{d} x}\right\|_{\left[0, R_{\alpha}\right]} \operatorname{area}_{\phi}(Q)\left\|P-P^{\prime}\right\|,
\end{aligned}
$$

where $\|g\|_{\left[0, R_{\alpha}\right]}$ denotes the $L_{\infty}$-norm of $g:\left[0, R_{\alpha}\right] \rightarrow \mathbb{R}$, and $\left\|P-P^{\prime}\right\|$ is the Euclidean norm of $P-P^{\prime}$ as a vector in $\mathbb{R}^{2 n}$. Here we have made use of the fact that, for all $q \in Q$, the map $P \mapsto \operatorname{dist}(q, P)$ is globally Lipschitz with Lipschitz constant 1. For $q \in S_{1}$, we have

$$
\begin{aligned}
\mid \int_{S_{1}}\left(f_{\alpha}(\operatorname{dist}(q, P))\right. & \left.1_{[0, R)}(\operatorname{dist}(q, P))-f_{\alpha}\left(\operatorname{dist}\left(q, P^{\prime}\right)\right) 1_{[0, R)}\left(\operatorname{dist}\left(q, P^{\prime}\right)\right)\right) \phi(q) \mathrm{d} q \mid \\
& \leq \int_{S_{1}}\left|f_{\alpha}(\operatorname{dist}(q, P))\right| \phi(q) \mathrm{d} q \leq\|\phi\|_{Q}\left\|f_{\alpha}\right\|_{\left[0, R_{\alpha}\right]} \int_{S_{1}} \mathrm{~d} q \\
& \leq\|\phi\|_{Q}\left\|f_{\alpha}\right\|_{\left[0, R_{\alpha}\right]} \sum_{i=1}^{n} \int_{\bar{B}\left(p_{i}, R\right) \cap\left(\cap_{j \in\{1, \ldots, n\}} \bar{B}\left(p_{j}^{\prime}, R\right)^{c}\right)} \mathrm{d} q \\
& \leq\|\phi\|_{Q}\left\|f_{\alpha}\right\|_{\left[0, R_{\alpha}\right]} \sum_{i=1}^{n} \int_{\bar{B}\left(p_{i}, R\right) \cap \bar{B}\left(p_{i}^{\prime}, R\right)^{c}} \mathrm{~d} q,
\end{aligned}
$$

where $\|\phi\|_{Q}=\max _{q \in Q} \phi(q)$. Now, we observe that, for $\left\|p_{i}-p_{i}^{\prime}\right\| \geq R$,

$$
\int_{\bar{B}\left(p_{i}, R\right) \cap \bar{B}\left(p_{i}^{\prime}, R\right)^{c}} \mathrm{~d} q \leq \pi R^{2} \leq \pi \operatorname{diam}(Q)\left\|p_{i}-p_{i}^{\prime}\right\| .
$$


On the other hand, for $\left\|p_{i}-p_{i}^{\prime}\right\| \leq R$, Lemma B.1 in Appendix B shows that

$$
\int_{\bar{B}\left(p_{i}, R\right) \cap \bar{B}\left(p_{i}^{\prime}, R\right)^{c}} \mathrm{~d} q \leq \frac{2 \sqrt{3}+3}{3} R\left\|p_{i}-p_{i}^{\prime}\right\| \leq \frac{2 \sqrt{3}+3}{3} \operatorname{diam}(Q)\left\|p_{i}-p_{i}^{\prime}\right\|
$$

Therefore, since $\frac{2 \sqrt{3}+3}{3}<\pi$, we have

$$
\begin{aligned}
& \left|\int_{S_{1}}\left(f_{\alpha}(\operatorname{dist}(q, P)) 1_{[0, R)}(\operatorname{dist}(q, P))-f_{\alpha}\left(\operatorname{dist}\left(q, P^{\prime}\right)\right) 1_{[0, R)}\left(\operatorname{dist}\left(q, P^{\prime}\right)\right)\right) \phi(q) \mathrm{d} q\right| \\
& \leq \pi\|\phi\|_{Q}\left\|f_{\alpha}\right\|_{\left[0, R_{\alpha}\right]} \operatorname{diam}(Q) \sum_{i=1}^{n}\left\|p_{i}-p_{i}^{\prime}\right\| \leq \frac{\pi}{\sqrt{n}}\|\phi\|_{Q}\left\|f_{\alpha}\right\|_{\left[0, R_{\alpha}\right]} \operatorname{diam}(Q)\left\|P-P^{\prime}\right\| .
\end{aligned}
$$

The integral over $S_{2}$ can be bounded in an analogous fashion. Summarizing, we have proved that $\mathcal{H}_{\alpha, R}$ satisfies

$$
\left|\mathcal{H}_{\alpha, R}(P)-\mathcal{H}_{\alpha, R}\left(P^{\prime}\right)\right| \leq L_{\alpha, R}\left\|P-P^{\prime}\right\|
$$

with $L_{\alpha, R}=\frac{2 \pi}{\sqrt{n}}\|\phi\|_{Q}\left\|f_{\alpha}\right\|_{\left[0, R_{\alpha}\right]} \operatorname{diam}(Q)+\left\|\frac{\mathrm{d} f_{\alpha}}{\mathrm{d} x}\right\|_{\left[0, R_{\alpha}\right]} \operatorname{area}_{\phi}(Q)$. This concludes the proof of the statement that $\mathcal{H}$ is globally Lipschitz on $Q^{n}$.

Next, we prove fact (ii), that is, we prove that $\mathcal{H}$ is continuously differentiable on $Q^{n} \backslash \mathcal{S}$ and we compute its partial derivative with respect to $p_{i}$. Consider the expression (6) for the function $\mathcal{H}$. Note that for each $i \in\{1, \ldots, n\}$ and $\alpha \in\{1, \ldots, m+1\}$, the function $(q, P) \mapsto f_{\alpha}\left(\left\|q-p_{i}\right\|\right)$ is continuous, and also continuously differentiable with respect to its second argument for all $P \in Q^{n}$ and almost all $q \in V_{i}(P) \cap D\left(p_{i},\left[R_{\alpha-1}, R_{\alpha}\right]\right)$. Note also that, for fixed $P \in Q^{n}$, both $q \mapsto f_{\alpha}\left(\left\|q-p_{i}\right\|\right)$ and $q \mapsto \frac{\partial}{\partial P}\left(f_{\alpha}\left(\left\|q-p_{i}\right\|\right)\right)$ are integrable on $V_{i}(P) \cap$ $D\left(p_{i},\left[R_{\alpha-1}, R_{\alpha}\right]\right)$. Furthermore, if $P \notin \mathcal{S}$, then the set

$$
\left\{q \in Q \mid \exists i, j \in\{1, \ldots, n\}, i \neq j, \text { such that }\left\|q-p_{i}\right\|=\left\|q-p_{j}\right\| \leq\left\|q-p_{k}\right\| \text { for } k \in\{1, \ldots, n\} \backslash\{i, j\}\right\}
$$

has measure zero. Therefore, $\left\{V_{i}(P) \mid P \in Q^{n} \backslash \mathcal{S}\right\}$ is a piecewise smooth family for each $i \in\{1, \ldots, n\}$. Since for each $\alpha \in\{1, \ldots, m+1\}$, the balls $\left\{\bar{B}\left(p_{i}, R_{\alpha}\right) \mid P \in Q^{n}\right\}$ also define a piecewise smooth family, one concludes that the intersection $V_{i} \cap D\left(p_{i},\left[R_{\alpha-1}, R_{\alpha}\right]\right)=V_{i} \cap \bar{B}\left(p_{i}, R_{\alpha}\right) \backslash V_{i} \cap B\left(p_{i}, R_{\alpha-1}\right)$, with $P \in Q^{n} \backslash \mathcal{S}$, can be written as the difference of two piecewise smooth families with strictly star-shaped sets. Applying now Proposition A.1 (see also Rem. A.2), we deduce that each summand in equation (6) is continuously differentiable on $Q^{n} \backslash \mathcal{S}$. We now compute its partial derivative with respect to $p_{i}, i \in\{1, \ldots, n\}$, as

$$
\begin{aligned}
\frac{\partial \mathcal{H}}{\partial p_{i}}(P)= & \frac{\partial}{\partial p_{i}}\left(\sum_{\alpha=1}^{m+1} \int_{V_{i}(P) \cap D\left(p_{i},\left[R_{\alpha-1}, R_{\alpha}\right]\right)} f_{\alpha}\left(\left\|q-p_{i}\right\|\right) \phi(q) \mathrm{d} q\right) \\
& +\frac{\partial}{\partial p_{i}}\left(\sum_{j \neq i} \sum_{\alpha=1}^{m+1} \int_{V_{j}(P) \cap D\left(p_{j},\left[R_{\alpha-1}, R_{\alpha}\right]\right)} f_{\alpha}\left(\left\|q-p_{j}\right\|\right) \phi(q) \mathrm{d} q\right)
\end{aligned}
$$


For each $k \in\{1, \ldots, n\}$ and each $\alpha \in\{1, \ldots, m+1\}$, let $n_{k, \alpha}(q)$ denote the unit outward normal to $V_{k}(P) \cap$ $\bar{B}\left(p_{k}, R_{\alpha}\right)$ at $q$, and let $\gamma_{k, \alpha}: \mathbb{S}^{1} \times Q^{n} \backslash \mathcal{S} \rightarrow \mathbb{R}^{2}$ denote a parameterization for the family $\left\{V_{k}(P) \cap \bar{B}\left(p_{k}, R_{\alpha}\right) \mid P \in\right.$ $\left.Q^{n} \backslash \mathcal{S}\right\}$. Using Proposition A.1, the above expression is equal to

$$
\begin{aligned}
\frac{\partial \mathcal{H}}{\partial p_{i}}(P)= & \sum_{\alpha=1}^{m+1} \int_{V_{i}(P) \cap D\left(p_{i},\left[R_{\alpha-1}, R_{\alpha}\right]\right)} \frac{\partial}{\partial p_{i}} f_{\alpha}\left(\left\|q-p_{i}\right\|\right) \phi(q) \mathrm{d} q \\
& +\sum_{\alpha=1}^{m+1} \int_{\partial\left(V_{i}(P) \cap \bar{B}\left(p_{i}, R_{\alpha}\right)\right)} n_{i, \alpha}^{t} \frac{\partial \gamma_{i, \alpha}}{\partial p_{i}} f_{\alpha}\left(\operatorname{dist}\left(\cdot, p_{i}\right)\right) \phi \\
& -\sum_{\alpha=1}^{m+1} \int_{\partial\left(V_{i}(P) \cap \bar{B}\left(p_{i}, R_{\alpha-1}\right)\right)} n_{i, \alpha-1}^{t} \frac{\partial \gamma_{i, \alpha-1}}{\partial p_{i}} f_{\alpha}\left(\operatorname{dist}\left(\cdot, p_{i}\right)\right) \phi \\
& +\sum_{\alpha=1}^{m+1} \sum_{j \neq i} \int_{\partial\left(V_{j}(P) \cap \bar{B}\left(p_{j}, R_{\alpha}\right)\right) \cap \partial\left(V_{i}(P) \cap \bar{B}\left(p_{i}, R_{\alpha}\right)\right)} n_{j, \alpha}^{t} \frac{\partial \gamma_{j, \alpha}}{\partial p_{i}} f_{\alpha}\left(\operatorname{dist}\left(\cdot, p_{j}\right)\right) \phi \\
& -\sum_{\alpha=1}^{m+1} \sum_{j \neq i} \int_{\partial\left(V_{j}(P) \cap \bar{B}\left(p_{j}, R_{\alpha-1}\right)\right) \cap \partial\left(V_{i}(P) \cap \bar{B}\left(p_{i}, R_{\alpha-1}\right)\right)} n_{j, \alpha-1}^{t} \frac{\partial \gamma_{j, \alpha-1}}{\partial p_{i}} f_{\alpha}\left(\operatorname{dist}\left(\cdot, p_{j}\right)\right) \phi
\end{aligned}
$$

where recall that $\operatorname{dist}(q, p)=\|q-p\|$. For $\alpha \in\{1, \ldots, m+1\}$, note that $\Delta_{i j}\left(2 R_{\alpha}\right)=\left(V_{i}(P) \cap \bar{B}\left(p_{i}, R_{\alpha}\right)\right) \cap$ $\left(V_{j}(P) \cap \bar{B}\left(p_{j}, R_{\alpha}\right)\right) \neq \emptyset$ if and only if $p_{i}$ and $p_{j}$ are neighbors according to the $2 R_{\alpha}$-limited Delaunay graph $\mathcal{G}_{\mathrm{LD}}\left(P, 2 R_{\alpha}\right)$. In this case, there exist intervals $\left[\theta_{i, j}^{-}(P), \theta_{i, j}^{+}(P)\right]$ and $\left[\theta_{j, i}^{-}(P), \theta_{j, i}^{+}(P)\right]$ depending smoothly on $P$ over an open set of $Q^{n} \backslash \mathcal{S}$ such that

$$
\theta \in\left[\theta_{i, j}^{-}(P), \theta_{i, j}^{+}(P)\right] \mapsto \gamma_{i, \alpha}(\theta, P), \quad \bar{\theta} \in\left[\theta_{j, i}^{-}(P), \theta_{j, i}^{+}(P)\right] \mapsto \gamma_{j, \alpha}(\bar{\theta}, P),
$$

are two parameterizations of the set $\left(V_{i}(P) \cap \bar{B}\left(p_{i}, R_{\alpha}\right)\right) \cap\left(V_{j}(P) \cap \bar{B}\left(p_{j}, R_{\alpha}\right)\right)$. Resorting to the implicit function theorem, one can show that there exists a function $h: \mathbb{S}^{1} \times U \rightarrow \mathbb{S}^{1}, h\left(\left[\theta_{j, i}^{-}(P), \theta_{j, i}^{+}(P)\right], P\right)=\left[\theta_{i, j}^{-}(P), \theta_{i, j}^{+}(P)\right]$, such that $\gamma_{j, \alpha}(\theta, P)=\gamma_{i, \alpha}(h(\theta, P), P)$ for $\theta \in\left[\theta_{j, i}^{-}(P), \theta_{j, i}^{+}(P)\right]$. From here, we deduce that $n_{j, \alpha}^{t} \frac{\partial \gamma_{j, \alpha}}{\partial p_{i}}=$ $n_{j, \alpha}^{t}\left(\frac{\partial \gamma_{i, \alpha}}{\partial \theta} \frac{\partial h}{\partial p_{i}}+\frac{\partial \gamma_{i, \alpha}}{\partial p_{i}}\right)=n_{j, \alpha}^{t} \frac{\partial \gamma_{i, \alpha}}{\partial p_{i}}$, since $n_{j, \alpha}$ and $\frac{\partial \gamma_{i, \alpha}}{\partial \theta}$ are orthogonal. Therefore, if $p_{j} \in \mathcal{N}_{\mathcal{G}_{\mathrm{LD}}\left(P, 2 R_{\alpha}\right)}\left(p_{i}\right)$, we have

$$
\begin{aligned}
& \int_{\partial\left(V_{j}(P) \cap \bar{B}\left(p_{j}, R_{\alpha}\right) \cap V_{i}(P) \cap \bar{B}\left(p_{i}, R_{\alpha}\right)\right)} n_{j, \alpha}^{t} \frac{\partial \gamma_{j, \alpha}}{\partial p_{i}} f_{\alpha}\left(\operatorname{dist}\left(\cdot, p_{j}\right)\right) \phi \\
&=-\int_{\partial\left(V_{i}(P) \cap \bar{B}\left(p_{i}, R_{\alpha}\right) \cap V_{j}(P) \cap \bar{B}\left(p_{j}, R_{\alpha}\right)\right)} n_{i, \alpha}^{t} \frac{\partial \gamma_{i, \alpha}}{\partial p_{i}} f_{\alpha}\left(\operatorname{dist}\left(\cdot, p_{i}\right)\right) \phi,
\end{aligned}
$$

since $n_{i, \alpha}(q)=-n_{j, \alpha}(q)$ and $\left\|q-p_{i}\right\|=\left\|q-p_{j}\right\|$ for all $q \in \partial\left(V_{i}(P) \cap \bar{B}\left(p_{i}, R_{\alpha}\right) \cap V_{j}(P) \cap \bar{B}\left(p_{j}, R_{\alpha}\right)\right)$. Moreover, notice that if $p_{i}$ moves, the motion - projected to the normal direction $n_{i, \alpha}$ - of the points in the $\operatorname{arcs}\left\{\operatorname{arc}_{i, 1}\left(2 R_{\alpha}\right), \ldots, \operatorname{arc}_{i, M_{i}\left(2 R_{\alpha}\right)}\left(2 R_{\alpha}\right)\right\} \subset \partial\left(V_{i}(P) \cap \bar{B}\left(p_{i}, R_{\alpha}\right)\right)$ is exactly the same as the motion of $p_{i}$, i.e., $n_{i, \alpha}^{t} \frac{\partial \gamma_{i, \alpha}}{\partial p_{i}}=n_{i, \alpha}^{t}$ over $\operatorname{arc}_{i, 1}\left(2 R_{\alpha}\right) \cup \cdots \cup \operatorname{arc}_{i, M_{i}\left(2 R_{\alpha}\right)}\left(2 R_{\alpha}\right)$. Using this fact, the expression for the partial 
derivative of $\mathcal{H}$ with respect to $p_{i}$ can be rewritten as

$$
\begin{array}{r}
\frac{\partial \mathcal{H}}{\partial p_{i}}(P)=\int_{V_{i}(P)} \frac{\partial}{\partial p_{i}} f\left(\left\|q-p_{i}\right\|\right) \phi(q) \mathrm{d} q+\sum_{\alpha=1}^{m+1}\left(\sum_{k=1}^{M_{i}\left(2 R_{\alpha}\right)} \int_{\operatorname{arc}_{i, k}\left(2 R_{\alpha}\right)} n_{\bar{B}\left(p_{i}, R_{\alpha}\right)} f_{\alpha}\left(R_{\alpha}\right) \phi\right. \\
\left.-\sum_{k=1}^{M_{i}\left(2 R_{\alpha-1}\right)} \int_{\operatorname{arc}_{i, k}\left(2 R_{\alpha-1}\right)} n_{\bar{B}\left(p_{i}, R_{\alpha-1}\right)} f_{\alpha}\left(R_{\alpha-1}\right) \phi\right)
\end{array}
$$

The final result is a rearrangement of the terms in this equation.

Remark 2.3. For a constant density function, $q \mapsto \phi(q)=c \in \overline{\mathbb{R}}_{+}$, each line integral

$$
\int_{\operatorname{arc}(2 R)} n \bar{B}(p, R) \phi
$$

computed over the $\operatorname{arc}(2 R)$ described by $\left[\theta_{-}, \theta_{+}\right] \ni \theta \mapsto p+R(\cos \theta, \sin \theta) \in \mathbb{R}^{2}$, equals

$$
c R \int_{\theta_{-}}^{\theta_{+}}(\cos \theta, \sin \theta) \mathrm{d} \theta=2 c R \sin \left(\frac{\theta_{+}-\theta_{-}}{2}\right)\left(\cos \left(\frac{\theta_{+}+\theta_{-}}{2}\right), \sin \left(\frac{\theta_{+}+\theta_{-}}{2}\right)\right) .
$$

For particular choices of performance function, the corresponding multi-center function and its gradient have different features. We here explore some interesting cases:

Centroid problem. If the performance function $f$ is piecewise differentiable with no jump discontinuities, then all the terms in the second summand of equation (7) vanish and one obtains

$$
\frac{\partial \mathcal{H}}{\partial p_{i}}(P)=\int_{V_{i}(P)} \frac{\partial}{\partial p_{i}} f\left(\left\|q-p_{i}\right\|\right) \phi(q) \mathrm{d} q .
$$

This is the result known in the locational optimization literature [2,11,23]. In particular, if $f(x)=-x^{2}$, the multi-center function $\mathcal{H}$ reads

$$
\mathcal{H}(P)=-\sum_{i=1}^{n} \int_{V_{i}(P)}\left\|q-p_{i}\right\|^{2} \phi(q) \mathrm{d} q \triangleq-\sum_{i=1}^{n} J_{V_{i}, p_{i}}
$$

where $J_{W, p}$ denotes the polar moment of inertia of the set $W \subset Q$ about the point $p$. Additionally, the gradient of $\mathcal{H}$ is

$$
\frac{\partial \mathcal{H}}{\partial p_{i}}(P)=2 \int_{V_{i}(P)}\left(q-p_{i}\right) \phi(q) \mathrm{d} q=2 \mathrm{M}_{V_{i}(P)}\left(\mathrm{CM}_{V_{i}(P)}-p_{i}\right)
$$

Here $\mathrm{M}_{W}$ and $\mathrm{CM}_{W}$ denote, respectively, the mass and the center of mass with respect to the density function $\phi$ of the set $W \subset Q$. The critical points of $\mathcal{H}$ are configurations $P \in Q^{n}$ such that $p_{i}=\mathrm{CM}_{V_{i}(P)}$ for all $i \in\{1, \ldots, n\}$. Such configurations are called centroidal Voronoi configurations, see [11].

Area problem. On the other hand, if one takes $f(x)=1_{[0, R]}(x)$, the indicator function of the set $[0, R]$, then the multi-center function $\mathcal{H}$ corresponds to the area, measured according to $\phi$, covered by the union of the $n$ balls $\bar{B}\left(p_{1}, R\right), \ldots, \bar{B}\left(p_{n}, R\right)$, that is,

$$
\mathcal{H}(P)=\operatorname{area}_{\phi}\left(\cup_{i=1}^{n} \bar{B}\left(p_{i}, R\right)\right) .
$$


In this case, the first term in equation (7) vanishes and one obtains

$$
\frac{\partial \mathcal{H}}{\partial p_{i}}(P)=\sum_{k=1}^{M_{i}(2 R)} \int_{\operatorname{arc}_{i, k}(2 R)} n_{\bar{B}\left(p_{i}, R\right)} \phi
$$

Given a configuration $P \in Q^{n}$, if the $i$ th agent is surrounded by neighbors in the graph $\mathcal{G}_{\mathrm{LD}}(P, 2 R)$ in such a way that $M_{i}(2 R)=0$, then the multi-center function $\mathcal{H}$ does not depend on $p_{i}$. This situation is depicted in Figure 3 (see example on the right) and captures the fact that the total area covered by the agents is not affected by an infinitesimal displacement of the $i$ th agent.

Mixed centroid-area problem. Consider the case when the function $f$ is given by $x \mapsto$ $-x^{2} 1_{[0, R)}(x)+b \cdot 1_{[R,+\infty)}(x)$, for $b \leq-R^{2}$. The multi-center function then takes the form

$$
\mathcal{H}(P)=-\sum_{i=1}^{n} J_{V_{i}(P) \cap \bar{B}\left(p_{i}, R\right), p_{i}}+b \operatorname{area}_{\phi}\left(Q \backslash \cup_{i=1}^{n} \bar{B}\left(p_{i}, R\right)\right),
$$

and its partial derivative with respect to the position of the $i$ th agent is

$$
\frac{\partial \mathcal{H}}{\partial p_{i}}(P)=2 \mathrm{M}_{V_{i}(P) \cap \bar{B}\left(p_{i}, R\right)}\left(\mathrm{CM}_{V_{i}(P) \cap \bar{B}\left(p_{i}, R\right)}-p_{i}\right)-\left(R^{2}+b\right) \sum_{k=1}^{M_{i}(2 R)} \int_{\operatorname{arc}_{i, k}(2 R)} n_{\bar{B}\left(p_{i}, R\right)} \phi
$$

In the particular case when $b=-R^{2}$, the function $x \mapsto f(x)=-x^{2} 1_{[0, R)}(x)-R^{2} \cdot 1_{[R,+\infty)}(x)$ is continuous and therefore the gradient of $\mathcal{H}$ takes the form

$$
\frac{\partial \mathcal{H}}{\partial p_{i}}(P)=2 \int_{V_{i}(P) \cap \bar{B}\left(p_{i}, R\right)}\left(q-p_{i}\right) \phi(q) \mathrm{d} q=2 \mathrm{M}_{V_{i}(P) \cap \bar{B}\left(p_{i}, R\right)}\left(\mathrm{CM}_{V_{i}(P) \cap \bar{B}\left(p_{i}, R\right)}-p_{i}\right) .
$$

Note that, in this case, the critical points of $\mathcal{H}$ are configurations $P \in Q^{n}$ such that $p_{i}=\mathrm{CM}_{V_{i}(P) \cap \bar{B}\left(p_{i}, R\right)}$ for all $i \in\{1, \ldots, n\}$. We refer to such configurations as $R$-centroidal Voronoi configurations. For $R \geq \operatorname{diam}(Q), R$-centroidal Voronoi configurations coincide with the standard centroidal Voronoi configurations over $Q$.

We can now characterize the results in Theorem 2.2 in terms of the notion of spatially-distributed computations introduced in Section 1.2.

Corollary 2.4. Let $\phi$ and $f$ be a density and a performance function, respectively. The gradient of $\mathcal{H}$ with respect to the agents' location $P \in Q^{n}$ is spatially distributed over the Delaunay graph $\mathcal{G}_{\mathrm{D}}(P)$. Furthermore, if $f(x)=b$ for all $x \geq R$, then the gradient of $\mathcal{H}$ with respect to the agents' location is spatially distributed over the $2 R$-limited Delaunay graph $\mathcal{G}_{\mathrm{LD}}(P, 2 R)$.

Proof. In general, the partial derivative of $\mathcal{H}$ with respect to the $i$ th agent ( $c f$. equation (7)) depends on the position $p_{i}$ and on the position of all neighbors of $p_{i}$ in the graph $\mathcal{G}_{\mathrm{D}}$. If, in addition, $f(x)=b$, for all $x \geq R$, then necessarily $R_{\alpha}<R, \alpha \in\{1, \ldots, m\}$, and

$$
\int_{V_{i}(P)} \frac{\partial}{\partial p_{i}} f\left(\left\|q-p_{i}\right\|\right) \phi(q) \mathrm{d} q=\int_{V_{i}(P) \cap \bar{B}\left(p_{i}, R\right)} \frac{\partial}{\partial p_{i}} f\left(\left\|q-p_{i}\right\|\right) \phi(q) \mathrm{d} q .
$$

Therefore, the expression for $\partial \mathcal{H} / \partial p_{i}$ in equation (7) can be computed with the knowledge $p_{i}$ and of its neighbors in the graph $\mathcal{G}_{\mathrm{LD}}(P, 2 R)$. 
This corollary states that information about all neighbors in $\mathcal{G}_{\mathrm{D}}$ is required for objective functions $\mathcal{H}$ corresponding to arbitrary performance functions $f$. In the next proposition we explore what can be done with only information about the neighbors in the $2 R$-limited Delaunay graph $\mathcal{G}_{\mathrm{LD}}(2 R)$.

Proposition 2.5. Let $f$ be a performance function and, without loss of generality, assume $f(0)=0$. For $r \in] 0,2 \operatorname{diam}(Q)]$, define the performance function $f_{\frac{r}{2}}: \overline{\mathbb{R}}_{+} \rightarrow \mathbb{R}$ given by $f_{\frac{r}{2}}(x)=f(x)$ for $x<\frac{r}{2}$ and $f_{\frac{r}{2}}(x)=f(\operatorname{diam}(Q))$ for $x \geq \frac{r}{2}$. Let $\mathcal{H}_{\frac{r}{2}}$ be the multi-center function associated to the performance function $f_{\frac{r}{2}}$. Then, for all $P \in Q^{n}$,

$$
\begin{aligned}
& \mathcal{H}_{\frac{r}{2}}(P) \leq \mathcal{H}(P) \leq \beta \mathcal{H}_{\frac{r}{2}}(P)<0, \\
& \mathcal{H}_{\frac{r}{2}}(P) \leq \mathcal{H}(P) \leq \mathcal{H}_{\frac{r}{2}}(P)+\Pi(P),
\end{aligned}
$$

where $\beta=\frac{f\left(\frac{r}{2}\right)}{f(\operatorname{diam}(Q))} \in[0,1]$ and $\Pi: Q^{n} \rightarrow[0, \kappa] \subset \mathbb{R}, \Pi(P)=\left(f\left(\frac{r}{2}\right)-f(\operatorname{diam}(Q))\right) \operatorname{area}_{\phi}\left(Q \backslash \cup_{i=1}^{n} \bar{B}\left(p_{i}, \frac{r}{2}\right)\right)$, with $\kappa=\left(f\left(\frac{r}{2}\right)-f(\operatorname{diam}(Q))\right) \operatorname{area}_{\phi}(Q)$.

Proof. Clearly, $f_{\frac{r}{2}}$ is a performance function as it is non-increasing and piecewise differentiable with finite jump discontinuities. Let $b=f(\operatorname{diam}(Q))$ and note that $f(x) \geq b$ for all $x \in[0, \operatorname{diam}(Q)]$. By construction, it is clear that $f_{\frac{r}{2}}(x) \leq f(x)$ for all $x \in[0, \operatorname{diam}(Q)]$. Since $\|q-p\| \leq \operatorname{diam}(Q)$ for all $q, p \in Q$, we conclude that $\mathcal{H}_{\frac{r}{2}}(P) \leq \mathcal{H}(P)$. Now, consider the function $\tilde{f}(x)=\beta f_{\frac{r}{2}}(x)$. Note that $\tilde{f}(x)=\beta f(x) \geq f(x)$ for $x<\frac{r}{2}$, and $\tilde{f}(x)=\beta b=f\left(\frac{r}{2}\right) \geq f(x)$ for $x \geq \frac{r}{2}$. Therefore,

$$
\beta \mathcal{H}_{\frac{r}{2}}(P)=\int_{Q} \max _{i \in\{1, \ldots, n\}} \tilde{f}\left(\left\|q-p_{i}\right\|\right) \phi(q) \mathrm{d} q \geq \int_{Q} \max _{i \in\{1, \ldots, n\}} f\left(\left\|q-p_{i}\right\|\right) \phi(q) \mathrm{d} q=\mathcal{H}(P),
$$

which concludes the proof of the first chain of inequalities. To prove the second chain of inequalities, consider the difference

$$
\mathcal{H}(P)-\mathcal{H}_{\frac{r}{2}}(P)=\sum_{i=1}^{n} \int_{V_{i}(P) \cap\left(Q \backslash \bar{B}\left(p_{i}, \frac{r}{2}\right)\right)}\left(f\left(\left\|q-p_{i}\right\|\right)-b\right) \phi(q) \mathrm{d} q .
$$

For $q \in V_{i}(P) \cap\left(Q \backslash \bar{B}\left(p_{i}, \frac{r}{2}\right)\right)$, the non-increasing property of $f$ implies that $f\left(\left\|q-p_{i}\right\|\right)-b \leq f\left(\frac{r}{2}\right)-b$. Therefore,

$$
\mathcal{H}(P)-\mathcal{H}_{\frac{r}{2}}(P) \leq \sum_{i=1}^{n} \int_{V_{i}(P) \cap\left(Q \backslash \bar{B}\left(p_{i}, \frac{r}{2}\right)\right)}\left(f\left(\frac{r}{2}\right)-b\right) \phi(q) \mathrm{d} q=\int_{Q \backslash \cup_{i=1}^{n} \bar{B}\left(p_{i}, \frac{r}{2}\right)}\left(f\left(\frac{r}{2}\right)-b\right) \phi(q) \mathrm{d} q=\Pi(P) .
$$

Remark 2.6. The inequalities in (10) provide, respectively, constant-factor and additive approximations of the value of the multi-center function $\mathcal{H}$ by the value of the function $\mathcal{H}_{\frac{r}{2}}$. These approximations will play an important role in Section 3 when we discuss the continuous and discrete-time implementations of spatiallydistributed coordination algorithms.

The next result provides one more useful indication of the relationship between multi-center functions associated to certain performance functions.

Proposition 2.7. Let $f$ and $f_{\frac{r}{2}}$ be performance functions, and $\mathcal{H}$ and $\mathcal{H}_{\frac{r}{2}}$ be the corresponding multi-center functions, defined as in Proposition 2.5. Let $P^{*}=\left(p_{1}^{*}, \ldots, p_{n}^{*}\right) \in Q^{n}$ be a local maximum of $\mathcal{H}_{\frac{r}{2}}$ such that $Q \subset$ $\cup_{i \in\{1, \ldots, n\}} \bar{B}\left(p_{i}^{*}, \frac{r}{2}\right)$. Then $\mathcal{H}\left(P^{*}\right)=\mathcal{H}_{\frac{r}{2}}\left(P^{*}\right)$ and $P^{*}$ is a local maximum of the aggregate objective function $\mathcal{H}$.

Proof. If $Q \subset \cup_{i \in\{1, \ldots, n\}} \bar{B}\left(p_{i}^{*}, \frac{r}{2}\right)$, then from equation (10b) we deduce that $\mathcal{H}\left(P^{*}\right)=\mathcal{H}_{\frac{r}{2}}\left(P^{*}\right)$. Moreover, one can also show that $V_{i}(P) \subset \bar{B}\left(p_{i}^{*}, \frac{r}{2}\right)$ for all $i \in\{1, \ldots, n\}$, and therefore $V_{i}(P)=V_{i}(P) \cap \bar{B}\left(p_{i}^{*}, \frac{r}{2}\right)$. As a consequence, the $r$-limited Delaunay graph $\mathcal{G}_{\mathrm{LD}}\left(P^{*}, r\right)$ and the Delaunay graph $\mathcal{G}_{\mathrm{D}}\left(P^{*}\right)$ coincide, and the gradients of both $\mathcal{H}$ and $\mathcal{H}_{\frac{r}{2}}$ vanish at $P^{*}$. 
The importance of Proposition 2.7 lies in the fact that, by following the gradient of the function $\mathcal{H}_{\frac{r}{2}}$ (where, along the evolution, the inclusion $Q \subset \cup_{i \in\{1, \ldots, n\}} \bar{B}\left(p_{i}, \frac{r}{2}\right)$ may not be verified and each agent only operates with the knowledge of (i) the positions of other agents up to a distance $r$ of its own position, and (ii) the events taking place at up to distance $\frac{r}{2}$ of its own position), the agents may eventually find a local maximum of the original multi-center function $\mathcal{H}$.

We end this section by presenting a useful result in the 1-center case, i.e., when there is a single agent $(n=1)$. For a convex polygon $W$, define the function $\mathcal{H}_{1}(p, W)=\int_{W} f(\|q-p\|) \phi(q) \mathrm{d} q$. The following lemma proves that the points in the boundary of $W$ are not local maxima of $\mathcal{H}_{1}(\cdot, W): W \rightarrow \mathbb{R}$.

Lemma 2.8. Let $W$ be a convex polygon, and consider the function $\mathcal{H}_{1}(\cdot, W): W \rightarrow \mathbb{R}$. Let $p_{0} \in \partial W$. Then the gradient of $\mathcal{H}_{1}$ at $p_{0}$ is non-vanishing $\frac{\partial \mathcal{H}_{1}(\cdot, W)}{\partial p}\left(p_{0}\right) \neq 0$, and points toward $\operatorname{int}(W)$.

Proof. The function $p \mapsto \mathcal{H}_{1}(p, W)$ is continuously differentiable over $W$, and its derivative is given by

$$
\begin{aligned}
\frac{\partial \mathcal{H}_{1}(\cdot, W)}{\partial p}= & \int_{W} \frac{\partial}{\partial p} f(\|q-p\|) \phi(q) \mathrm{d} q+\sum_{\alpha=1}^{m+1} \int_{\partial\left(W \cap \bar{B}\left(p, R_{\alpha}\right)\right)} n_{\alpha}^{t} \frac{\partial \gamma_{\alpha}}{\partial p} f_{\alpha}(\operatorname{dist}(\cdot, p)) \phi \\
& -\sum_{\alpha=1}^{m+1} \int_{\partial\left(W \cap \bar{B}\left(p, R_{\alpha-1}\right)\right)} n_{\alpha-1}^{t} \frac{\partial \gamma_{\alpha-1}}{\partial p} f_{\alpha}(\operatorname{dist}(\cdot, p)) \phi .
\end{aligned}
$$

Let $M\left(R_{\alpha}\right) \geq 0$ denote the number of distinct $\operatorname{arcs} \operatorname{arc}_{1}\left(2 R_{\alpha}\right), \ldots, \operatorname{arc}_{M\left(R_{\alpha}\right)}\left(2 R_{\alpha}\right)$ of radius $R_{\alpha}$ in $\partial(W \cap$ $\left.\bar{B}\left(p, R_{\alpha}\right)\right)$. After some simplifications, we rewrite the expression for the gradient at $p_{0}$ as

$$
-\int_{W} f^{\prime}\left(\left\|q-p_{0}\right\|\right) \frac{q-p_{0}}{\left\|q-p_{0}\right\|} \phi(q) \mathrm{d} q+\sum_{\alpha=1}^{m}\left(f_{\alpha}\left(R_{\alpha}\right)-f_{\alpha+1}\left(R_{\alpha}\right)\right) \sum_{l=1}^{M\left(R_{\alpha}\right)} \int_{\operatorname{arc}_{l}\left(2 R_{\alpha}\right)} n_{\alpha} \phi
$$

where $n_{\alpha}$ denotes the outward normal to $\bar{B}\left(p_{0}, R_{\alpha}\right)$. Since $W$ is convex, it is defined as the intersection of half spaces with supporting hyperplanes $H_{1}, \ldots, H_{d}$ via the equations $H_{\zeta}(q)=A_{\zeta} q+b_{\zeta} \geq 0$, where $A_{\zeta}$ is a $2 \times 2$-matrix and $b_{\zeta} \in \mathbb{R}$, for $\zeta \in\{1, \ldots, d\}$. To show that $\frac{\partial \mathcal{H}_{1}(\cdot, W)}{\partial p}\left(p_{0}\right) \neq 0$ and points $\operatorname{toward} \operatorname{int}(W)$, we consider its inner product with the direction given by the each line $A_{\zeta_{*}} q+b_{\zeta_{*}}=0$ such that $H_{\zeta_{*}}\left(p_{0}\right)=0$. Let us therefore consider

$$
\begin{gathered}
A_{\zeta_{*}}\left(-\int_{W} f^{\prime}\left(\left\|q-p_{0}\right\|\right) \frac{q-p_{0}}{\left\|q-p_{0}\right\|} \phi(q) \mathrm{d} q+\sum_{\alpha=1}^{m}\left(f_{\alpha}\left(R_{\alpha}\right)-f_{\alpha+1}\left(R_{\alpha}\right)\right) \sum_{l=1}^{M\left(R_{\alpha}\right)} \int_{\operatorname{arc}_{l}\left(2 R_{\alpha}\right)} n_{\alpha} \phi\right) \\
=-\int_{W} f^{\prime}\left(\left\|q-p_{0}\right\|\right) \frac{A_{\zeta_{*}} q+b_{\zeta_{*}}}{\left\|q-p_{0}\right\|} \phi(q) \mathrm{d} q+\sum_{\alpha=1}^{m}\left(f_{\alpha}\left(R_{\alpha}\right)-f_{\alpha+1}\left(R_{\alpha}\right)\right) \sum_{l=1}^{M\left(R_{\alpha}\right)} \int_{\operatorname{arc}_{l}\left(2 R_{\alpha}\right)} \frac{A_{\zeta_{*}}(\cdot)+b_{\zeta_{*}}}{\operatorname{dist}\left(\cdot, p_{0}\right)} \phi
\end{gathered}
$$

where we have used the fact that $n_{\alpha}(q)=\left(q-p_{0}\right) /\left\|q-p_{0}\right\|$ for each $q \in \partial\left(W \cap \bar{B}\left(p_{0}, R_{\alpha}\right)\right)$. Since the function $f$ is non-increasing, then its derivative is negative almost everywhere, and the jump discontinuities $f_{\alpha}\left(R_{\alpha}\right)-f_{\alpha+1}\left(R_{\alpha}\right)$ are positive for all $\alpha \in\{1, \ldots, m\}$. Finally, note that $A_{\zeta_{*}} q+b_{\zeta_{*}}>0$ in the interior of $W$. Therefore, we conclude that $A_{\zeta_{*}}\left(\frac{\partial \mathcal{H}_{1}(\cdot, W)}{\partial p}\left(p_{0}\right)\right)>0$ for all $\zeta_{*}$ such that $H_{\zeta_{*}}\left(p_{0}\right)=0$, i.e., $\frac{\partial \mathcal{H}_{1}(\cdot, W)}{\partial p}\left(p_{0}\right) \neq 0$ and points toward $\operatorname{int}(W)$.

\section{Design of SPATIALly-Distributed Algorithms FOR COVERAGE CONTROL}

In this section, we develop continuous and discrete-time implementations of the gradient ascent for a general aggregate objective function $\mathcal{H}$. 


\subsection{Continuous-time implementations}

Assume the agents' location obeys a first order dynamical behavior described by

$$
\dot{p}_{i}=u_{i}
$$

Consider $\mathcal{H}$ an aggregate objective function to be maximized and impose that the location $p_{i}$ follows the gradient ascent given by (7). In more precise terms, we set up the following control law defined over the set $Q^{n} \backslash \mathcal{S}$

$$
u_{i}=\frac{\partial \mathcal{H}}{\partial p_{i}}(P)
$$

where we assume that the partition $\mathcal{V}(P)=\left\{V_{1}, \ldots, V_{n}\right\}$ is continuously updated. One can prove the following result.

Proposition 3.1 (continuous-time Lloyd ascent). Consider the gradient vector field on $Q^{n} \backslash \mathcal{S}$ defined by equation (12). Then

(i) For a general performance function $f$, the gradient vector field is spatially distributed over the Delaunay graph $\mathcal{G}_{\mathrm{D}}(\mathcal{P})$. If, in addition, the performance function verifies $f(x)=b$ for all $x \geq R$, then the vector field is spatially distributed over the $2 R$-limited Delaunay graph $\mathcal{G}_{\mathrm{LD}}(P, 2 R)$.

(ii) The agents' location evolving under (12) starting at $P_{0} \in Q^{n} \backslash \mathcal{S}$ remains in $Q^{n} \backslash \mathcal{S}$ and converges asymptotically to the set of critical points of the aggregate objective function $\mathcal{H}$. Assuming this set is finite, the agents' location converges to a critical point of $\mathcal{H}$.

Proof. The statement (i) is a transcription of Corollary 2.4. To prove the statement (ii), let $t \in \overline{\mathbb{R}}_{+} \mapsto P(t) \in Q^{n}$ denote the solution to the initial value problem $\dot{p}_{i}=\frac{\partial \mathcal{H}_{\frac{r}{2}}}{\partial p_{i}}(P), i \in\{1, \ldots, n\}, P(0)=P_{0}$. We reason by contradiction. Assume that there exists $t_{*} \in \mathbb{R}_{+}$and $i, j \in\{1, \ldots, n\}$ such that $p_{i}\left(t_{*}\right)=p_{j}\left(t_{*}\right)$, i.e., $P\left(t_{*}\right) \in \mathcal{S}$. Let $v$ be an accumulation point of the sequence of unitary vectors $\left\{\frac{p_{i}(t)-p_{j}(t)}{\left\|p_{i}(t)-p_{j}(t)\right\|}\right\}_{t \rightarrow t_{*}^{-}}$. Let $\epsilon>0$ sufficiently small such that, for all $t \in\left(t_{*}-\epsilon, t_{*}\right), p_{i}(t)$ and $p_{j}(t)$ are neighbors in the graph $\mathcal{G}_{\mathrm{LD}}(P(t), r)$. Then one can show that

$$
v \cdot \frac{\partial \mathcal{H}_{\frac{r}{2}}}{\partial p_{i}}\left(P\left(t_{*}\right)\right)>0, \quad v \cdot \frac{\partial \mathcal{H}_{\frac{r}{2}}}{\partial p_{j}}\left(P\left(t_{*}\right)\right)<0 .
$$

Indeed, if $n$ denotes the orthogonal line to $v$, and $H_{i, n}$ and $H_{j, n}$ denote the associated hyperplanes having $v$ pointing inward and outward respectively, then, reasoning as in the proof of Lemma 2.8, one proves that $\frac{\partial \mathcal{H}_{\frac{r}{2}}}{\partial p_{i}}\left(P\left(t_{*}\right)\right)=\lim _{t \rightarrow t_{*}^{-}} \frac{\partial \mathcal{H}_{\frac{r}{2}}}{\partial p_{i}}(P(t))$ points toward $\operatorname{int}\left(V_{i}\left(P\left(t_{*}\right)\right) \cap \bar{B}\left(p_{i}\left(t_{*}\right), \frac{r}{2}\right) \cap H_{i, n}\right)$, and $\frac{\partial \mathcal{H}_{\frac{r}{2}}}{\partial p_{j}}\left(P\left(t_{*}\right)\right)=$ $\lim _{t \rightarrow t_{*}^{-}} \frac{\partial \mathcal{H}_{\frac{r}{2}}}{\partial p_{j}}(P(t))$ points toward $\operatorname{int}\left(V_{j}\left(P\left(t_{*}\right)\right) \cap \bar{B}\left(p_{j}\left(t_{*}\right), \frac{r}{2}\right) \cap H_{j, n}\right)$. Since equation (13) is valid for any accumulation point of the sequence $\left\{\frac{p_{i}(t)-p_{j}(t)}{\left\|p_{i}(t)-p_{j}(t)\right\|}\right\}_{t \rightarrow t_{*}^{-}}$, we deduce that for all $t$ sufficiently close to $t_{*}$, we have $\left(p_{i}(t)-p_{j}(t)\right) \cdot\left(\dot{p}_{i}(t)-\dot{p}_{j}(t)\right)>0$, which contradicts $P\left(t_{*}\right) \in \mathcal{S}$. One can resort to a similar argument to guarantee that there is no configuration belonging to $\mathcal{S}$ in the $\omega$-limit set of the curve $t \mapsto P(t)$. The convergence result to the set of critical points of $\mathcal{H}_{\frac{r}{2}}$ is an application of LaSalle Invariance Principle [18].

Remark 3.2. Note that this gradient ascent is not guaranteed to find the global maximum. For example, in the vector quantization and signal processing literature [14], it is known that for "bimodal" distribution density functions, the solution to the gradient flow reaches local maxima where the number of agents allocated to the two region of maxima are not optimally partitioned.

In a practical setting, the sensing and/or communication capabilities of a network agent are restricted to a bounded region specified by a finite radius $r>0$. Therefore, instead of maximizing the multi-center function 
$\mathcal{H}$, we set up the continuous-time algorithm given by equation (12) with the function $\mathcal{H}_{\frac{r}{2}}$. This latter algorithm has the advantage of being spatially distributed over the $r$-limited Delaunay graph $\mathcal{G}_{\mathrm{LD}}(P, r)$, and providing an approximation of the behavior for the multi-center function $\mathcal{H}$ ( $c f$. Prop. 2.5).

\subsection{Discrete-time implementations}

We start by reviewing some notions on discrete-time algorithms following [21]. An algorithm on $Q^{n}$ is a set-valued map $T: Q^{n} \rightarrow 2^{Q^{n}}$. Note that a map from $Q^{n}$ to $Q^{n}$ can be interpreted as a singleton-valued map. For any initial $P_{0} \in Q^{n}$, an algorithm $T$ generates feasible sequences of configurations in the following way: given $P_{n} \in Q^{n}$, the map $T$ yields $T\left(P_{n}\right) \subset Q^{n}$. From this set, an arbitrary element $P_{n+1}$ may be selected. In other words,

$$
P_{n+1} \in T\left(P_{n}\right), \quad n \in \mathbb{N} \cup\{0\} .
$$

An algorithm $T$ is said to be closed at $P \in Q^{n}$ (cf. [21]) if for all convergent sequences $P_{k} \rightarrow P, P_{k}^{\prime} \rightarrow P^{\prime}$ such that $P_{k}^{\prime} \in T\left(P_{k}\right)$, one has that $P^{\prime} \in T(P)$. An algorithm is said to be closed on $W \subset Q^{n}$ if it is closed at $P$, for all $P \in W$. In particular, every continuous map $T: Q^{n} \rightarrow Q^{n}$ is closed on $Q^{n}$. A set $C$ is said to be weakly positively invariant with respect to $T$ if for any $P_{0} \in C$ there exists $P \in T\left(P_{0}\right)$ such that $P \in C$. A point $P_{*}$ is said to be a fixed point of $T$ if $P_{*} \in T\left(P_{*}\right)$. Let $U: Q^{n} \rightarrow \mathbb{R}$. We say that $U$ is a Lyapunov function for $T$ on $W$ if (i) $U$ is continuous on $W$ and (ii) $U\left(P^{\prime}\right) \leq U(P)$ for all $P^{\prime} \in T(P)$ and all $P \in W$.

We now turn to the design of discrete-time algorithms for limited-range coverage control. We start by extending the definition of the aggregate objective function $\mathcal{H}$ to consider general partitions $\mathcal{W}$ of $Q$ as follows. Let $P \in Q^{n}$ and let $\mathcal{W}=\left\{W_{i} \subset Q\right\}_{i=1}^{n}$ be a partition of $Q$ such that $W_{i}$ is a convex polygon and $p_{i} \in W_{i}$, for $i \in\{1, \ldots, n\}$. Define the function

$$
\mathcal{H}_{e}(P, \mathcal{W})=\sum_{i=1}^{n} \int_{W_{i}} f\left(\left\|q-p_{i}\right\|\right) \phi(q) \mathrm{d} q
$$

The function $\mathcal{H}_{e}$ is continuously differentiable with respect to its first variable for all $P \in Q^{n}$, and its partial derivative is given by

$$
\begin{aligned}
\frac{\partial \mathcal{H}_{e}}{\partial p_{i}}(P, \mathcal{W})= & \sum_{\alpha=1}^{m+1} \int_{W_{i} \cap D\left(p_{i},\left[R_{\alpha-1}, R_{\alpha}\right]\right)} \frac{\partial}{\partial p_{i}} f_{\alpha}\left(\left\|q-p_{i}\right\|\right) \phi(q) \mathrm{d} q \\
& +\sum_{\alpha=1}^{m+1} \int_{\partial\left(W_{i} \cap \bar{B}\left(p_{i}, R_{\alpha}\right)\right)} n_{i, \alpha}^{t} \frac{\partial \gamma_{i, \alpha}}{\partial p_{i}} f_{\alpha}\left(\operatorname{dist}\left(\cdot, p_{i}\right)\right) \phi \\
& -\sum_{\alpha=1}^{m+1} \int_{\partial\left(W_{i} \cap \bar{B}\left(p_{i}, R_{\alpha-1}\right)\right)} n_{i, \alpha-1}^{t} \frac{\partial \gamma_{i, \alpha-1}}{\partial p_{i}} f_{\alpha}\left(\operatorname{dist}\left(\cdot, p_{i}\right)\right) \phi
\end{aligned}
$$

where for each $k \in\{1, \ldots, n\}$ and each $\alpha \in\{1, \ldots, m+1\}, n_{k, \alpha}(q)$ denotes the unit outward normal to $W_{k} \cap \bar{B}\left(p_{k}, R_{\alpha}\right)$ at $q$, and $\gamma_{k, \alpha}: \mathbb{S}^{1} \times Q^{n} \rightarrow \mathbb{R}^{2}$ denotes a parameterization for the piecewise smooth family $\left\{W_{k} \cap \bar{B}\left(p_{k}, R_{\alpha}\right) \mid P \in Q^{n}\right\}$. Note that, using the definition of $\mathcal{H}_{1}$ (cf. Sect. 2), one can also write

$$
\mathcal{H}_{e}(P, \mathcal{W})=\sum_{i=1}^{n} \mathcal{H}_{1}\left(p_{i}, W_{i}\right)
$$


The following two equalities hold

$$
\begin{aligned}
\mathcal{H}(P) & =\mathcal{H}_{e}(P, \mathcal{V}(P)), \quad \text { for all } P \in Q^{n}, \\
\frac{\partial \mathcal{H}_{e}}{\partial p_{i}}(P, \mathcal{V}(P)) & =\frac{\partial \mathcal{H}}{\partial p_{i}}(P), \quad \text { for all } P \in Q^{n} \backslash \mathcal{S}
\end{aligned}
$$

Let $P \in \mathcal{S}$ and consider a partition $\mathcal{W}=\left\{W_{i} \subset Q\right\}_{i=1}^{n}$ of $Q$ such that $W_{i}$ is a convex polygon and $p_{i} \in W_{i}$, for $i \in\{1, \ldots, n\}$. Let $i_{0}, j_{0} \in\{1, \ldots, n\}, i_{0} \neq j_{0}$ such that $p_{i_{0}}=p_{j_{0}}$. Then, following Remark $1.3, V_{i_{0}}(P)=V_{j_{0}}(P)$, and $\mathcal{V}(P)$ is no longer a partition of $Q$, but a covering. Nevertheless, one could consider the line determined by the edge $W_{i_{0}} \cap W_{j_{0}}$ and the associated hyperplanes $H_{i_{0}, W_{i_{0}} \cap W_{j_{0}}}$ and $H_{j_{0}, W_{i_{0}} \cap W_{j_{0}}}$ such that $W_{i_{0}} \subset H_{i_{0}, W_{i_{0}} \cap W_{j_{0}}}$ and $W_{j_{0}} \subset H_{j_{0}, W_{i_{0}} \cap W_{j_{0}}}$. With a slight abuse of notation, redefining

$$
V_{i_{0}}(P)=V_{i_{0}}(P) \cap H_{i_{0}, W_{i_{0}} \cap W_{j_{0}}}, \quad V_{j_{0}}(P)=V_{j_{0}}(P) \cap H_{j_{0}, W_{i_{0}} \cap W_{j_{0}}},
$$

the collection $\mathcal{V}(P)$ can be seen a partition of $Q$. This procedure can be extended if there are more than two coincident agents $\left\{i_{1}, \ldots, i_{s}\right\}$ at a point $p \in Q$ by defining

$$
V_{i_{\mu}}(P)=V_{i_{\mu}}(P) \cap\left(\cap_{\nu \in\{1, \ldots, s\} \backslash\{\mu\}} H_{i_{\mu}, W_{i_{\mu}} \cap W_{i_{\nu}}}\right), \quad \mu \in\{1, \ldots, s\} .
$$

In the following, such a construction will be tacitly performed whenever we have a configuration $P \in \mathcal{S}$ and a partition $\mathcal{W}$ of $Q$.

The following lemma shows that the Voronoi partition is optimal within the set of partitions of $Q$.

Lemma 3.3. Let $\phi$ and $f$ be a density and a performance function, respectively. Let $P \in Q^{n}$ and consider a partition $\mathcal{W}=\left\{W_{i} \subset Q\right\}_{i=1}^{n}$ of $Q$ such that $W_{i}$ is a convex polygon and $p_{i} \in W_{i}$, for $i \in\{1, \ldots, n\}$. Then

$$
\mathcal{H}_{e}(P, \mathcal{W}) \leq \mathcal{H}_{e}(P, \mathcal{V}(P))
$$

and the inequality is strict if $f$ is strictly decreasing and the partitions $\mathcal{V}(P)$ and $\mathcal{W}$ differ by a set of non-zero measure.

Proof. Given the chain of implications $q \in V_{j}(P) \Rightarrow\left\|q-p_{i}\right\| \geq\left\|q-p_{j}\right\| \Rightarrow f\left(\left\|q-p_{i}\right\|\right) \phi(q) \leq f\left(\left\|q-p_{j}\right\|\right) \phi(q)$, we compute

$$
\begin{aligned}
\mathcal{H}_{e}(P, \mathcal{W}) & =\sum_{i=1}^{n} \sum_{j=1}^{n} \int_{W_{i} \cap V_{j}(P)} f\left(\left\|q-p_{i}\right\|\right) \phi(q) \mathrm{d} q \\
& \leq \sum_{i=1}^{n} \sum_{j=1}^{n} \int_{W_{i} \cap V_{j}(P)} f\left(\left\|q-p_{j}\right\|\right) \phi(q) \mathrm{d} q=\mathcal{H}_{e}(P, \mathcal{V}(P))
\end{aligned}
$$

We are now ready to characterize a class of algorithms with guaranteed convergence to the set of critical points of the aggregate objective function $\mathcal{H}$.

Proposition 3.4 (discrete-time ascent). Let $T: Q^{n} \rightarrow 2^{Q^{n}}$ be an algorithm closed on $Q^{n} \backslash \mathcal{S}$ satisfying the following properties:

(a) for all $P \in Q^{n}, T(P) \cap \mathcal{S}=\emptyset$;

(b) for all $P \in Q^{n} \backslash \mathcal{S}, P^{\prime} \in T(P)$ and $i \in\{1, \ldots, n\}, \mathcal{H}_{1}\left(p_{i}^{\prime}, V_{i}(P)\right) \geq \mathcal{H}_{1}\left(p_{i}, V_{i}(P)\right)$;

(c) for all $P \in \mathcal{S}$ and $P^{\prime} \in T(P), \mathcal{H}\left(P^{\prime}\right)>\mathcal{H}(P)$;

(d) if $P \in Q^{n} \backslash \mathcal{S}$ is not a critical point of $\mathcal{H}$, then for all $P^{\prime} \in T(P)$, there exists $j \in\{1, \ldots, n\}$ such that $\mathcal{H}_{1}\left(p_{j}^{\prime}, V_{j}(P)\right)>\mathcal{H}_{1}\left(p_{j}, V_{j}(P)\right)$.

Let $P_{0} \in Q^{n}$ denote the initial agents' location. Then, any sequence $\left\{P_{n} \mid n \in \mathbb{N} \cup\{0\}\right\}$ generated according to equation (14) converges to the set of critical points of $\mathcal{H}$. 
Proof. Consider $-\mathcal{H}: Q^{n} \rightarrow \mathbb{R}$ as a candidate Lyapunov function for the algorithm $T$ on $Q^{n} \backslash \mathcal{S}$. Because of Lemma 3.3, we have

$$
\mathcal{H}\left(P^{\prime}\right)=\mathcal{H}_{e}\left(P^{\prime}, \mathcal{V}\left(P^{\prime}\right)\right) \geq \mathcal{H}_{e}\left(P^{\prime}, \mathcal{V}(P)\right)
$$

for all $P^{\prime} \in T(P)$. In addition, because of property (b) of $T$, we also have

$$
\mathcal{H}_{e}\left(P^{\prime}, \mathcal{V}(P)\right) \geq \mathcal{H}_{e}(P, \mathcal{V}(P))=\mathcal{H}(P)
$$

for all $P^{\prime} \in T(P)$. Hence, $\mathcal{H}\left(P^{\prime}\right) \leq \mathcal{H}(P)$ for all $P^{\prime} \in T(P)$ and all $P \in Q^{n}$. Therefore, we deduce that $-\mathcal{H}$ is a Lyapunov function for the algorithm $T$. Let $P_{0} \in Q^{n} \backslash \mathcal{S}$ and consider a sequence $\left\{P_{n} \mid n \in \mathbb{N} \cup\{0\}\right\}$ generated according to equation (14). Because of property (a) of $T,\left\{P_{n} \mid n \in \mathbb{N} \cup\{0\}\right\}$ remains in $Q^{n} \backslash \mathcal{S} \subset Q^{n}$. Since $Q^{n}$ is compact, we conclude that the sequence is bounded. Now, the application of the discrete-time LaSalle Invariance Principle (see Appendix C, Th. C.1) guarantees that there exists $c \in \mathbb{R}$ such that $P_{n} \rightarrow M \cap \mathcal{H}^{-1}(c)$, where $M$ is the largest weakly positively invariant set contained in $\left\{P \in Q^{n} \mid \exists P^{\prime} \in T(P)\right.$ such that $\left.\mathcal{H}\left(P^{\prime}\right)=\mathcal{H}(P)\right\}$. Properties (c) and (d) of $T$ imply that $M$ must be contained in the set of critical points of $\mathcal{H}$. If $P_{0} \in \mathcal{S}$, the sequence $\left\{P_{n} \mid n \in \mathbb{N} \cup\{0\}\right\}$ can be equivalently described by $\left\{P_{0}\right\} \cup\left\{P_{n} \mid n \in \mathbb{N}\right\}$. Since $P_{1} \in Q^{n} \backslash \mathcal{S}$ by property (a) of $T$, the previous argument implies that the sequence converges to the set of critical points of $\mathcal{H}$.

In what follows, we devise a general algorithm $T: Q^{n} \rightarrow 2^{Q^{n}}$ verifying properties (a)-(d) in Proposition 3.4. We shall do so by designing a discrete-time version of the gradient ascent algorithm for continuous-time settings.

Recall that Lemma 2.8 asserts that if $p_{0} \in \partial W$, then $\frac{\partial \mathcal{H}_{1}(\cdot, W)}{\partial p}\left(p_{0}\right) \neq 0$ points toward the interior of $W$. If $p_{0} \in \operatorname{int}(W)$ is not a critical point, then one also has that $\frac{\partial \mathcal{H}_{1}(\cdot, W)}{\partial p}\left(p_{0}\right) \neq 0$. For both cases, there exists $\epsilon=\epsilon\left(p_{0}, W\right)>0$ such that the point $p_{\delta}$ defined by

$$
p_{\delta}=p_{0}+\delta \frac{\partial \mathcal{H}_{1}(\cdot, W)}{\partial p}\left(p_{0}\right) \in W
$$

has the property that $\mathcal{H}_{1}\left(p_{\delta}\right)>\mathcal{H}_{1}\left(p_{0}\right)$, for all $\delta \in(0, \epsilon)$, and $\mathcal{H}_{1}\left(p_{\epsilon}\right)=\mathcal{H}_{1}\left(p_{0}\right)$. As it is usually done in nonlinear programming [21], the computation of the step-size $\epsilon$ can be implemented numerically via a "line search". With this discussion in mind, let us define the line search algorithm $T_{\mathrm{ls}}: Q^{n} \rightarrow 2^{Q^{n}}$ as follows:

Given $P \in Q^{n}$, let $P^{\prime} \in T_{\mathrm{ls}}(P)$ if, for $i \in\{1, \ldots, n\}$ with the property that $p_{i} \neq p_{j}, j \in$ $\{1, \ldots, n\} \backslash\{i\}$,

$$
p_{i}^{\prime}=p_{i}+\delta \frac{\partial \mathcal{H}_{1}\left(\cdot, V_{i}(P)\right)}{\partial p}\left(p_{i}\right), \text { with } \delta \in\left[\frac{\epsilon\left(p_{i}, V_{i}(P)\right)}{3}, \frac{\epsilon\left(p_{i}, V_{i}(P)\right)}{2}\right],
$$

and, for each set $\left\{i_{1}, \ldots, i_{s}\right\}$ of coincident indexes at a point $p \in Q$,

$$
p_{i_{\mu}}^{\prime}=p_{i_{\mu}}+\delta \frac{\partial \mathcal{H}_{1}\left(\cdot, Y_{i_{\mu}}\right)}{\partial p}\left(p_{i_{\mu}}\right), \text { with } \delta \in\left[\frac{\epsilon\left(p_{i}, Y_{i}\right)}{3}, \frac{\epsilon\left(p_{i}, Y_{i}\right)}{2}\right],
$$

where $\left\{Y_{i_{1}}, \ldots, Y_{i_{s}}\right\}$ is a partition of $V_{i_{1}}(P)=\cdots=V_{i_{s}}(P)$ verifying $p \in Y_{i_{\mu}}$, for $\mu \in\{1, \ldots, s\}$.

Proposition 3.5. The algorithm $T_{\mathrm{ls}}: Q^{n} \rightarrow 2^{Q^{n}}$ defined by equations (18)-(19) is closed on $Q^{n} \backslash \mathcal{S}$, and verifies properties (a)-(d) in Proposition 3.4.

Proof. The fact that $T_{\mathrm{ls}}$ is closed on $Q^{n} \backslash \mathcal{S}$ follows from its definition and the continuous dependence of $\epsilon(p, V(P))$ on $P \in Q^{n} \backslash \mathcal{S}$. Regarding the properties in Proposition 3.4, consider the following discussion. Let $P \in Q^{n}$ and consider $P^{\prime} \in T_{\mathrm{ls}}(P)$. On the one hand, equation (18) and the definition of $\epsilon(p, V(P))$ implies that $p_{i}^{\prime} \in \operatorname{int}\left(V_{i}(P)\right)$ for each $i \in\{1, \ldots, n\}$ such that $p_{i} \neq p_{j}$ for all $j \in\{1, \ldots, n\} \backslash\{i\}$. On the other hand, equation (19) and Lemma 2.8 implies $p_{i_{\mu}}^{\prime} \in \operatorname{int}\left(Y_{i_{\mu}}\right)$. Therefore, we deduce that $P^{\prime} \notin \mathcal{S}$, and 
property (a) is verified. Using equation (18), one has that for all $P \in Q^{n} \backslash \mathcal{S}, P^{\prime} \in T_{\mathrm{ls}}(P)$ and all $i \in\{1, \ldots, n\}$, $\mathcal{H}_{1}\left(p_{i}^{\prime}, V_{i}(P)\right) \geq \mathcal{H}_{1}\left(p_{i}, V_{i}(P)\right)$, i.e., the algorithm $T_{\text {ls }}$ verifies property (b). With respect to property (c), let $P \in \mathcal{S}$. For simplicity, we only deal with the case when there exists $i, j \in\{1, \ldots, n\}, i \neq j$ such that $p_{i}=p_{j}$, and all other $p_{k} \neq p_{i}=p_{j}, k \in\{1, \ldots, n\} \backslash\{i, j\}$ are distinct among them (the cases with more degeneracies are treated analogously). Let $P^{\prime} \in T(P)$. According to equation (19), we have

$$
\mathcal{H}(P)=\sum_{k \in\{1, \ldots, n\} \backslash\{i, j\}} \mathcal{H}_{1}\left(p_{k}, V_{k}(P)\right)+\mathcal{H}_{1}\left(p_{i}, Y_{i}\right)+\mathcal{H}_{1}\left(p_{j}, Y_{j}\right),
$$

where $\left\{Y_{i}, Y_{j}\right\}$ is a partition of $V_{i}(P)=V_{j}(P)$ with $p_{i} \in Y_{i}$ and $p_{j} \in Y_{j}$. Since necessarily $p_{i} \in \partial Y_{i}$ and $p_{j} \in \partial Y_{j}$, Lemma 2.8 implies that $\mathcal{H}_{1}\left(p_{i}, Y_{i}\right)+\mathcal{H}_{1}\left(p_{j}, Y_{j}\right)<\mathcal{H}_{1}\left(p_{i}^{\prime}, Y_{i}\right)+\mathcal{H}_{1}\left(p_{j}^{\prime}, Y_{j}\right)$. Therefore, $\mathcal{H}(P)<\mathcal{H}\left(P^{\prime}\right)$, i.e., property (c) is verified by $T_{\mathrm{ls}}$. Finally, if $P \in Q^{n} \backslash \mathcal{S}$ is not a critical point of $\mathcal{H}$, then there must exist $i \in\{1, \ldots, n\}$ such that

$$
\frac{\partial \mathcal{H}}{\partial p_{i}}(P)=\frac{\partial \mathcal{H}_{e}}{\partial p_{i}}(P, \mathcal{V}(P)) \neq 0 .
$$

Equivalently, $p_{i}$ is not a critical point of $\mathcal{H}_{1}\left(\cdot, V_{i}(P)\right): V_{i}(P) \rightarrow \mathbb{R}$, and therefore $\epsilon\left(p_{i}, V_{i}(P)\right)>0$. By equation (18), we conclude that $\mathcal{H}_{1}\left(p_{i}^{\prime}, V_{i}(P)\right)>\mathcal{H}_{1}\left(p_{i}, V_{i}(P)\right)$ for all $P^{\prime} \in T_{\mathrm{ls}}(P)$, i.e., the algorithm $T_{\mathrm{ls}}$ verifies property (d).

Corollary 3.6. Consider the algorithm $T_{\mathrm{ls}}: Q^{n} \rightarrow 2^{Q^{n}}$ defined by equations (18)-(19). Then

(i) for a general performance function $f$, the algorithm $T_{\mathrm{ls}}$ is spatially distributed over the Delaunay graph $\mathcal{G}_{\mathrm{D}}(\mathcal{P})$. If, in addition, the performance function verifies $f(x)=b$ for all $x \geq R$, then $T_{\mathrm{ls}}$ is spatially distributed over the $2 R$-limited Delaunay graph $\mathcal{G}_{\mathrm{LD}}(P, 2 R)$;

(ii) the sequence of agents' locations generated by $T_{\mathrm{ls}}$ according to equation (14) starting at $P_{0} \in Q^{n}$, converges asymptotically to the set of critical points of the aggregate objective function $\mathcal{H}$.

Proof. The statement (i) is a direct consequence of Corollary 2.4. The convergence result is a consequence of Propositions 3.4 and 3.5 .

Remark 3.7. As we noticed in Section 3.1, in a practical setting, the network agents have typically a limited sensing/communication radius $r>0$, and therefore, following the result in Proposition 2.5, we seek to maximize the function $\mathcal{H}_{\frac{r}{2}}$.

In certain cases, it might be possible to construct specific algorithms tailored to the concrete aggregate objective function at hand. A relevant example of this situation is when the local maxima of the function $\mathcal{H}_{1}(\cdot, W)$ can be characterized for each fixed polygon $W$. With this discussion in mind, let us define the max algorithm $T_{\max }: Q^{n} \rightarrow 2^{Q^{n}}$ as follows:

For $P \in Q^{n} \backslash \mathcal{S}$, let

$$
T_{\max }(P)=\left\{P^{\prime} \in Q^{n} \mid p_{i}^{\prime} \text { is a local maximum of } \mathcal{H}_{1}\left(\cdot, V_{i}(P)\right) \text {, for } i \in\{1, \ldots, n\}\right\} .
$$

If $P \in \mathcal{S}$, for each set $\left\{i_{1}, \ldots, i_{s}\right\}$ of coincident indexes at a point $p \in Q$, let $p_{i_{\mu}}^{\prime}$ be a local maximum of $\mathcal{H}_{1}\left(\cdot, Y_{i_{\mu}}\right)$, where $\left\{Y_{i_{1}}, \ldots, Y_{i_{s}}\right\}$ is a partition of $V_{i_{1}}(P)=\cdots=V_{i_{s}}(P)$ verifying $p \in Y_{i_{\mu}}$, for $\mu \in\{1, \ldots, s\}$.

One can show that $T_{\max }$ is closed on $Q^{n} \backslash \mathcal{S}$ and verifies properties (a)-(d) in Proposition 3.4. As before, the algorithm $T_{\max }$ is spatially distributed over the Delaunay graph $\mathcal{G}_{\mathrm{D}}(\mathcal{P})$ and, if the performance function is $f_{\frac{r}{2}}$, then $T_{\max }$ is spatially distributed over the $r$-limited Delaunay graph $\mathcal{G}_{\mathrm{LD}}(\mathcal{P}, r)$.

It is worth noticing that Lemma 2.8 guarantees that the local maxima of $\mathcal{H}_{1}(\cdot, W)$ are not in the boundary of $W$, and therefore are contained in the set $\left\{p_{*} \in W \mid \frac{\partial \mathcal{H}_{1}(\cdot, W)}{\partial p}\left(p_{*}\right)=0\right\}$. Moreover, if $f$ is concave, then $\mathcal{H}_{1}$ is also concave, as stated in the following lemma. 
Lemma 3.8. If $f: \overline{\mathbb{R}}_{+} \rightarrow \mathbb{R}$ is concave, then $\mathcal{H}_{1}$ is concave.

Proof. For fixed $q \in Q$, the map $p \mapsto f(\|q-p\|) \phi(q)$ is concave; the integral with respect to $q$ of a map with this property is concave in $p$; see [5], Section 3.2.1.

As a consequence, the set of global maxima of $\mathcal{H}_{1}(\cdot, W)$ is compact, convex and characterized by the equation

$$
\frac{\partial \mathcal{H}_{1}(\cdot, W)}{\partial p}(p)=0
$$

In particular, these conditions are met in the centroid problem introduced in Section 2 , where $f(x)=-x^{2}$ is concave and the unique global minimum of $\mathcal{H}_{1}(\cdot, W)$ is the centroid $\mathrm{CM}_{W}$ of $W$. In this case, the algorithm $T_{\max }$ is precisely the Lloyd quantization algorithm $[8,11,14]$.

\section{Simulations}

To illustrate the performance of the coordination algorithms, we include some simulation results. The algorithms are implemented in Mathematica ${ }^{\circledR}$ as a library of routines and a main program running the simulation. We have developed a library of basic geometric routines in two and three dimensions. The resulting Mathematica ${ }^{\circledR}$ packages PlanGeom.m and SpatialGeom.m are freely available at http://ams.ucsc.edu/ $\sim$ jcortes/software.

The objective of a first routine is to compute the intersection of the bounded Voronoi cell $V_{i}$ with the ball $\bar{B}\left(p_{i}, \frac{r}{2}\right)$, for $i \in\{1, \ldots, n\}$, and to parameterize each set $V_{i} \cap \bar{B}\left(p_{i}, \frac{r}{2}\right)$ in polar coordinates. The objective of a second routine is to compute the surface integrals on these sets and the line integrals on their boundaries via the numerical integration routine NIntegrate. We paid careful attention to numerical accuracy issues in the computation of the Voronoi diagram and in the integration.

We show executions of the discrete-time algorithm $T_{\mathrm{ls}}$ (cf. Eqs. (18) and (19)) for the centroid problem, the area problem, the mixed centroid-area problem with continuous sensing performance, and the mixed centroidarea problem with discontinuous sensing performance in Figures 4-7, respectively. Measuring displacements in meters, we consider the domain $Q$ determined by the vertices

$$
\{(0,0),(2.125,0),(2.9325,1.5),(2.975,1.6),(2.9325,1.7),(2.295,2.1),(0.85,2.3),(0.17,1.2)\} \text {. }
$$

The diameter of this domain is $\operatorname{diam}(Q)=3.37796$. In all examples, the distribution density function $\phi$ is the sum of five Gaussian functions of the form $5 \exp \left(6\left(-\left(x-x_{\text {center }}\right)^{2}-\left(y-y_{\text {center }}\right)^{2}\right)\right)$ and is represented by means of its contour plot. The centers $\left(x_{\text {center }}, y_{\text {center }}\right)$ of the Gaussians are given, respectively, by $(2,0.25),(1,2.25)$, $(1.9,1.9),(2.35,1.25)$ and $(0.1,0.1)$. Measured with respect to $\phi$, the area of the domain is $\operatorname{area}_{\phi}(Q)=8.61656$. Unless otherwise noted, each agent operates with a finite sensing/communication radius equal to $r=0.45$.

The execution of the coordination algorithm in Figure 7 (with radius $r=0.45$, agent performance $f_{\frac{r}{2}}(x)=$ $-x^{2} 1_{\left[0, \frac{r}{2}\right)}(x)-\operatorname{diam}(Q)^{2} \cdot 1_{\left[\frac{r}{2},+\infty\right)}(x)$ and corresponding multi-center function $\left.\mathcal{H}_{\frac{r}{2}}\right)$ can be regarded as a limitedrange implementation of the gradient ascent of the multi-center function $\mathcal{H}$ corresponding to the agent performance $f(x)=-x^{2}$ (cf. Fig. 4); this performance function does not have any range limitation. According to Proposition 2.5, we compute

$$
\begin{aligned}
\beta & =\frac{f\left(\frac{r}{2}\right)}{f(\operatorname{diam}(Q))}=\frac{1}{4}\left(\frac{r}{\operatorname{diam}(Q)}\right)^{2} \approx 0.004437, \\
\Pi\left(P_{\text {final }}\right) & =\left(f\left(\frac{r}{2}\right)-f(\operatorname{diam}(Q))\right) \operatorname{area}_{\phi}\left(Q \backslash \cup_{i=1}^{n} \bar{B}\left(p_{i}, \frac{r}{2}\right)\right) \approx 26.5156,
\end{aligned}
$$

where $P_{\text {final }}$ denotes the final configuration in Figure 7. From the constant-factor approximation (10a) and the additive approximation (10b), the absolute error is guaranteed to be less than or equal to $\min \left\{(\beta-1) \mathcal{H}_{\frac{r}{2}}\left(P_{\text {final }}\right), \Pi\left(P_{\text {final }}\right)\right\} \approx 6.77282$. In order to compare the performance of this execution with 

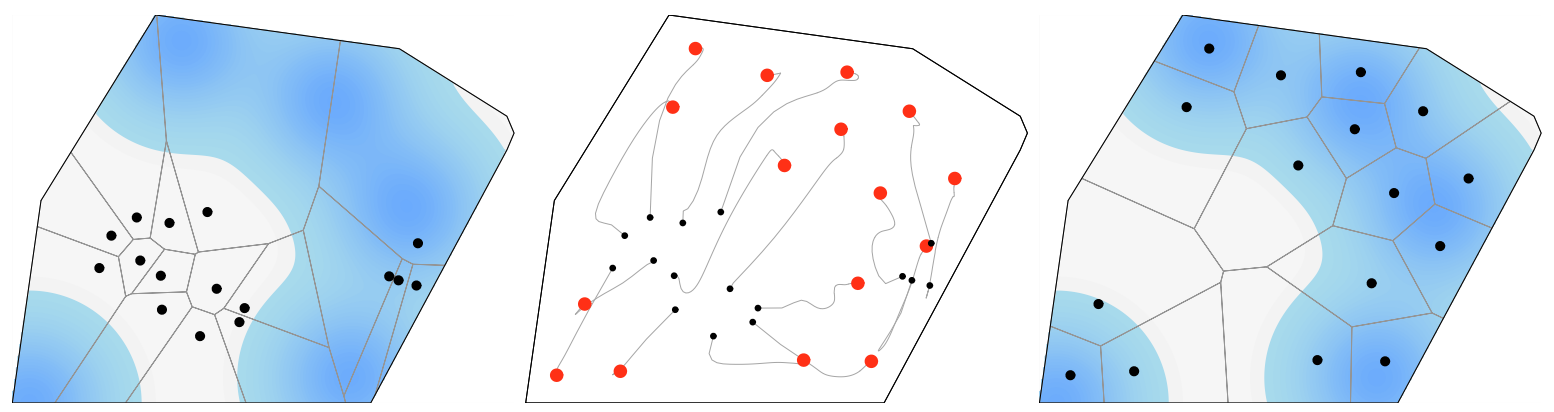

FIGURE 4. Centroid problem (with agent performance $f(x)=-x^{2}$ ): discrete-time algorithm $T_{\text {ls }}$ for 16 agents on a convex polygonal environment. The left (respectively, right) figure illustrates the initial (respectively, final) locations and Voronoi partition. The central figure illustrates the gradient ascent flow. After 90 seconds, the value of the multi-center function is approximately -0.321531 .
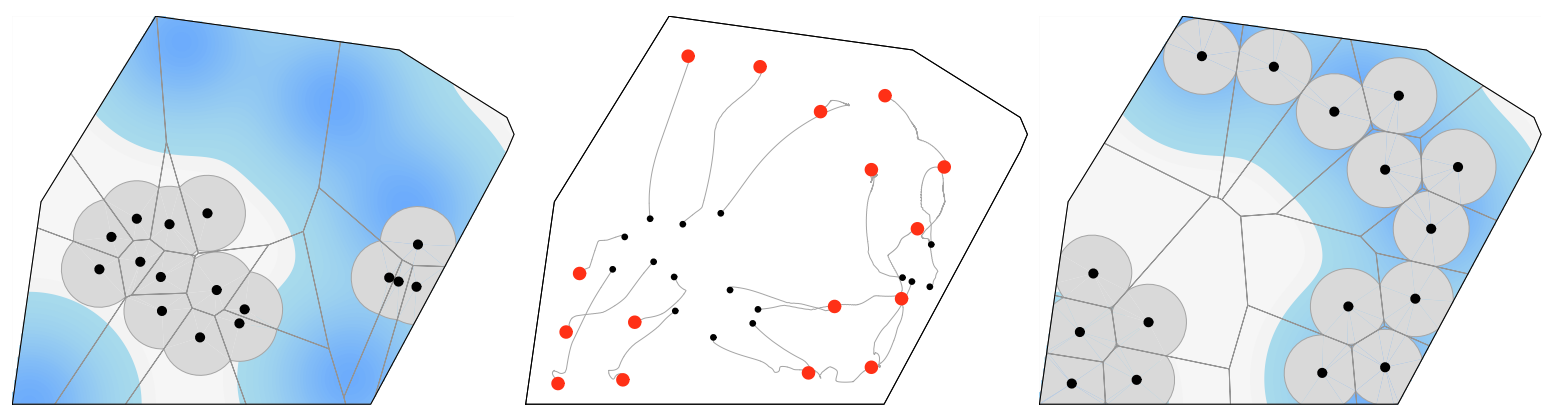

FIGURE 5. Area problem (with agent performance $f(x)=1_{\left[0, \frac{r}{2}\right]}(x)$ ): discrete-time algorithm $T_{\text {ls }}$ for 16 agents on a convex polygonal environment. The left (respectively, right) figure illustrates the initial (respectively, final) locations and Voronoi partition. The central figure illustrates the gradient ascent flow. For each agent $i$, the intersection $V_{i} \cap \bar{B}\left(p_{i}, \frac{r}{2}\right)$ is plotted in light gray. After 18 seconds, the value of the multi-center function is approximately 6.28977.
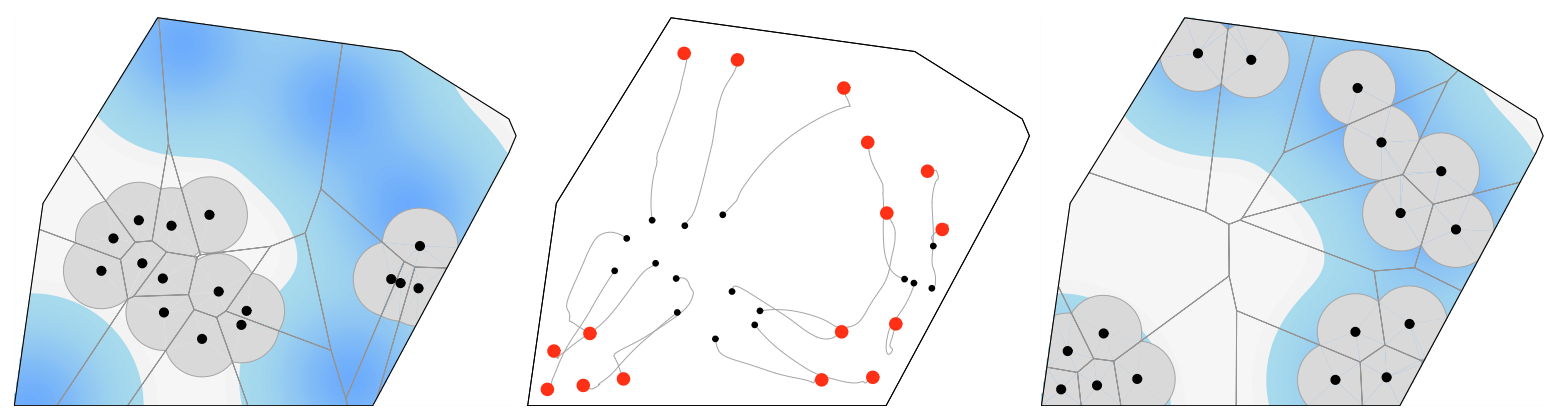

Figure 6. Mixed centroid-area problem (with continuous agent performance $f(x)=$ $\left.-x^{2} 1_{\left[0, \frac{r}{2}\right)}(x)-\frac{r^{2}}{4} \cdot 1_{\left[\frac{r}{2},+\infty\right)}(x)\right)$ : discrete-time algorithm $T_{\mathrm{ls}}$ for 16 agents on a convex polygonal environment. The left (respectively, right) figure illustrates the initial (respectively, final) locations and Voronoi partition. The central figure illustrates the gradient ascent flow. For each agent $i$, the intersection $V_{i} \cap \bar{B}\left(p_{i}, \frac{r}{2}\right)$ is plotted in light gray. After 90 seconds, the value of the multi-center function is approximately -0.252534 . 

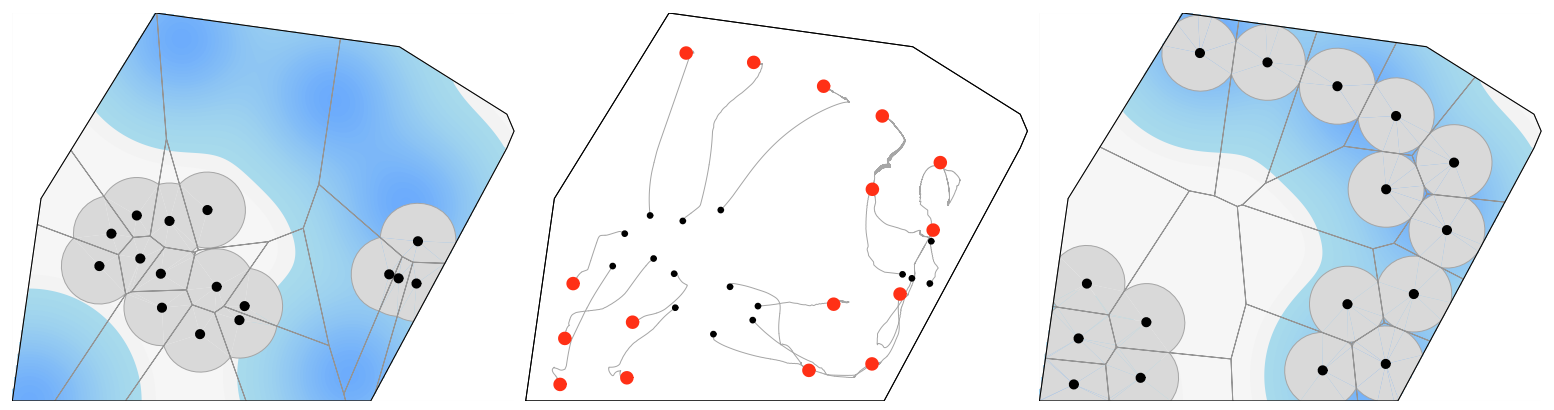

Figure 7. Mixed centroid-area problem (with discontinuous agent performance $f(x)=$ $\left.-x^{2} 1_{\left[0, \frac{r}{2}\right)}(x)-\operatorname{diam}(Q)^{2} \cdot 1_{\left[\frac{r}{2},+\infty\right)}(x)\right)$ : discrete-time algorithm $T_{\mathrm{ls}}$ for 16 agents on a convex polygonal environment. The left (respectively, right) figure illustrates the initial (respectively, final) locations and Voronoi partition. The central figure illustrates the gradient ascent flow. For each agent $i$, the intersection $V_{i} \cap \bar{B}\left(p_{i}, \frac{r}{2}\right)$ is plotted in light gray. After 13.5 seconds, the value of the multi-center function is approximately -6.803 .
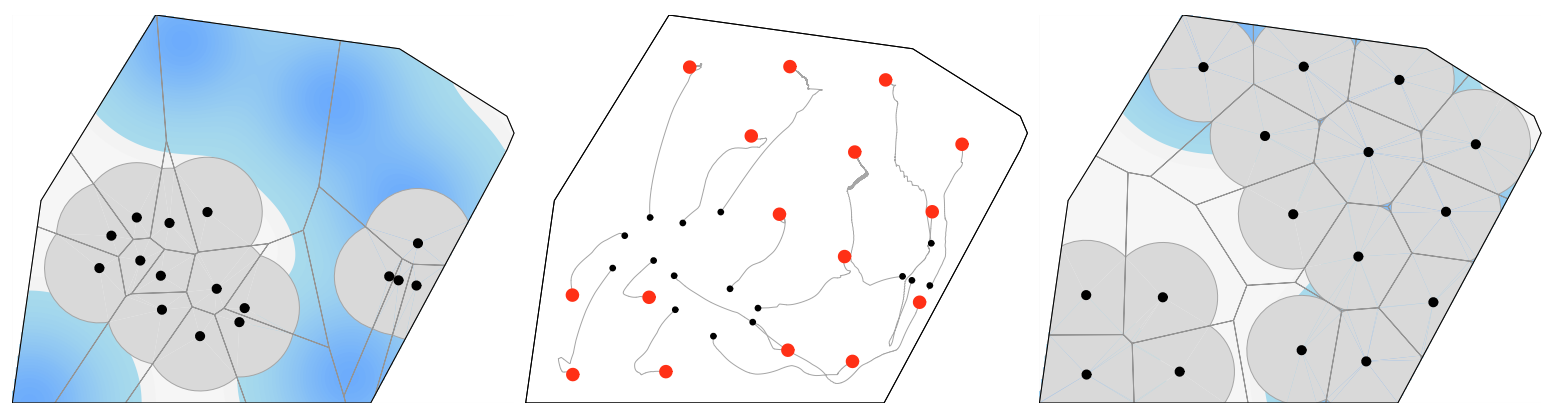

FiguRE 8. Execution of the discrete-time algorithm $T_{\mathrm{ls}}$ in the same setting as in Figure 7, but with a sensing/communication radius equal to $r=0.65$. After 13.5 seconds, the value of the multi-center function is approximately -1.10561 .

the performance of the discrete-time algorithm in the unlimited-range case, i.e., for the case of $f(x)=-x^{2}(c f$. Fig. 4), we compute the percentage error in the value of the multi-center function $\mathcal{H}$ at their final configurations. This percentage error is approximately equal to $30.7 \%$.

Figure 8 below shows another execution of the discrete-time algorithm $T_{\mathrm{ls}}$ for the mixed centroid-area problem with discontinuous sensing performance, where now the sensing/communication radius is taken equal to $r=0.65$. In this case, the percentage error with respect to the performance of the discrete-time algorithm in the unlimitedrange case is approximately equal to $23 \%$. As expected, the percentage error of the performance of the limitedrange implementation improves with higher values of the ratio $\frac{r}{\operatorname{diam}(Q)}$.

\section{Conclusions And Future WORK}

We have presented novel spatially-distributed algorithms for coordinated motion of groups of agents in continuous and discrete time. Avenues of possible future research include (1) distributed implementation of deterministic annealing techniques [29] (methods which promise to overcome local maxima), (2) visibilitybased algorithms for coverage in non-convex environments, and (3) distributed algorithms for other cooperative behaviors and sensing tasks, e.g., detection, estimation, and map-building. 
Acknowledgements. This material is based upon work supported in part by ARO Grant DAAD 190110716, ONR YIP Award N00014-03-1-0512, and NSF SENSORS Award IIS-0330008. Sonia Martínez's work was supported in part by a Fulbright PostDoctoral Fellowship from the Spanish Ministry of Education and Culture.

\section{A. Piecewise smooth Sets and a Generalized Conservation-OF-Mass LaW}

A set $S \subset \mathbb{R}^{2}$ is called strictly star-shaped if there exists a point $p \in S$ such that for all $s \in \partial S$ and all $\lambda \in(0,1]$, one has that $\lambda p+(1-\lambda) s \in \operatorname{int}(S)$. A curve $C$ in $\mathbb{R}^{2}$ is the image of a map $\gamma:[a, b] \rightarrow \mathbb{R}^{2}$. The map $\gamma$ is called a parameterization of $C$. A curve $\gamma:[a, b] \rightarrow \mathbb{R}^{2}$ is simple if it is not self-intersecting, i.e., if $\gamma$ is injective on $(a, b)$. A curve is closed if $\gamma(a)=\gamma(b)$. A set $\Omega \subset \mathbb{R}^{2}$ is said to be piecewise smooth if its boundary, $\partial \Omega$, is a simple closed curve that admits a continuous and piecewise smooth (i.e. piecewise continuously differentiable) parameterization $\gamma: \mathbb{S}^{1} \rightarrow \mathbb{R}^{2}$. Likewise, a collection of sets $\left\{\Omega(x) \subset \mathbb{R}^{2} \mid x \in(a, b)\right\}$ is said to be a piecewise smooth family if $\Omega(x)$ is piecewise smooth for all $x \in(a, b)$, and there exists a continuous function $\gamma: \mathbb{S}^{1} \times(a, b) \rightarrow \mathbb{R}^{2},(\theta, x) \mapsto \gamma(\theta, x)$, continuously differentiable with respect to its second argument such that for each $x \in(a, b)$, the map $\theta \mapsto \gamma_{x}(\theta)=\gamma(\theta, x)$ is a continuous and piecewise smooth parameterization of $\partial \Omega(x)$. We refer to $\gamma$ as a parameterization for the family $\left\{\Omega(x) \subset \mathbb{R}^{2} \mid x \in(a, b)\right\}$.

The following result is an extension of the integral form of the Conservation-of-Mass Law in fluid mechanics [6]. Given a curve $C$ parameterized by a piecewise smooth map $\gamma:[a, b] \rightarrow C$, recall that the line integral of a function $f: C \subset \mathbb{R}^{2} \rightarrow \mathbb{R}$ over $C$ is defined by

$$
\int_{C} f=\int_{a}^{b} f(\gamma(t))\|\dot{\gamma}(t)\| \mathrm{d} t
$$

and it is independent of the selected parameterization.

Proposition A.1. Let $\left\{\Omega(x) \subset \mathbb{R}^{2} \mid x \in(a, b)\right\}$ be a piecewise smooth family such that $\Omega(x)$ is strictly starshaped for all $x \in(a, b)$. Let the function $\varphi: \mathbb{R}^{2} \times(a, b) \rightarrow \mathbb{R}$ be continuous on $\mathbb{R}^{2} \times(a, b)$, continuously differentiable with respect to its second argument for all $x \in(a, b)$ and almost all $q \in \Omega(x)$, and such that for each $x \in(a, b)$, the maps $q \mapsto \varphi(q, x)$ and $q \mapsto \frac{\partial \varphi}{\partial x}(q, x)$ are measurable, and integrable on $\Omega(x)$. Then, the function

$$
(a, b) \ni x \mapsto \int_{\Omega(x)} \varphi(q, x) \mathrm{d} q
$$

is continuously differentiable and

$$
\frac{\mathrm{d}}{\mathrm{d} x} \int_{\Omega(x)} \varphi(q, x) \mathrm{d} q=\int_{\Omega(x)} \frac{\partial \varphi}{\partial x}(q, x) \mathrm{d} q+\int_{\partial \Omega(x)} \varphi(\gamma, x) n^{t}(\gamma) \frac{\partial \gamma}{\partial x} \mathrm{~d} \gamma
$$

where $n: \partial \Omega(x) \rightarrow \mathbb{R}^{2}, q \mapsto n(q)$, denotes the unit outward normal to $\partial \Omega(x)$ at $q \in \partial \Omega(x)$, and $\gamma: \mathbb{S}^{1} \times(a, b) \rightarrow$ $\mathbb{R}^{2}$ is a parameterization for the family $\left\{\Omega(x) \subset \mathbb{R}^{2} \mid x \in(a, b)\right\}$.

Proof. Let $x_{0} \in(a, b)$. Using the fact that the map $\gamma$ is continuous and that $\Omega\left(x_{0}\right)$ is strictly star-shaped, one can show that there exist an interval around $x_{0}$ of the form $\mathcal{I}_{x_{0}}=\left(x_{0}-\epsilon, x_{0}+\epsilon\right)$, a continuously differentiable function $u_{x_{0}}: \mathbb{S}^{1} \times \overline{\mathbb{R}}_{+} \rightarrow \mathbb{R}^{2}$ and a function $r_{x_{0}}: \mathbb{S}^{1} \times \mathcal{I}_{x_{0}} \rightarrow \overline{\mathbb{R}}_{+}$continuously differentiable in $x$ and piecewise continuously differentiable in $\theta$ such that for all $x \in \mathcal{I}_{x_{0}}$, one has $\Omega(x)=\cup_{\theta \in \mathbb{S}^{1}}\left\{u_{x_{0}}(\theta, s) \mid 0 \leq s \leq r_{x_{0}}(\theta, x)\right\}$ and $u_{x_{0}}\left(\theta, r_{x_{0}}(\theta, x)\right)=\gamma(\theta, x)$, for $\theta \in \mathbb{S}^{1}$. For simplicity, we denote by $r$ and $u$ the functions $r_{x_{0}}$ and $u_{x_{0}}$, respectively. By definition, the function in (21) is continuously differentiable at $x_{0}$ if the following limit exists

$$
\lim _{h \rightarrow 0} \frac{1}{h}\left(\int_{\Omega\left(x_{0}+h\right)} \varphi\left(q, x_{0}+h\right) \mathrm{d} q-\int_{\Omega\left(x_{0}\right)} \varphi\left(q, x_{0}\right) \mathrm{d} q\right),
$$


and depends continuously on $x_{0}$. Now, we can rewrite the previous limit as

$$
\begin{aligned}
\lim _{h \rightarrow 0} \frac{1}{h} \int_{0}^{2 \pi}\left(\int_{0}^{r\left(\theta, x_{0}+h\right)} \varphi\left(u(\theta, s), x_{0}+h\right)\left\|\frac{\partial u}{\partial \theta} \times \frac{\partial u}{\partial s}\right\| \mathrm{d} s-\int_{0}^{r\left(\theta, x_{0}\right)} \varphi\left(u(\theta, s), x_{0}\right)\left\|\frac{\partial u}{\partial \theta} \times \frac{\partial u}{\partial s}\right\| \mathrm{d} s\right) \mathrm{d} \theta= \\
\lim _{h \rightarrow 0} \frac{1}{h} \int_{0}^{2 \pi}\left(\int_{r\left(\theta, x_{0}\right)}^{r\left(\theta, x_{0}+h\right)} \varphi\left(u(\theta, s), x_{0}+h\right)\left\|\frac{\partial u}{\partial \theta} \times \frac{\partial u}{\partial s}\right\| \mathrm{d} s\right. \\
\left.\quad+\int_{0}^{r\left(\theta, x_{0}\right)}\left(\varphi\left(u(\theta, s), x_{0}+h\right)-\varphi\left(u(\theta, s), x_{0}\right)\right)\left\|\frac{\partial u}{\partial \theta} \times \frac{\partial u}{\partial s}\right\| \mathrm{d} s\right) \mathrm{d} \theta
\end{aligned}
$$

where $\times$ denotes the vector product and for brevity we omit that the partial derivatives $\frac{\partial u}{\partial \theta}$ and $\frac{\partial u}{\partial s}$ are evaluated at $(\theta, s)$ in the integrals. Now, since

$$
\lim _{h \rightarrow 0} \frac{1}{h}\left(\varphi\left(u(\theta, s), x_{0}+h\right)-\varphi\left(u(\theta, s), x_{0}\right)\left\|\frac{\partial u}{\partial \theta} \times \frac{\partial u}{\partial s}\right\|\right)=\frac{\partial \varphi}{\partial x_{0}}\left(u(\theta, s), x_{0}\right)\left\|\frac{\partial u}{\partial \theta} \times \frac{\partial u}{\partial s}\right\|
$$

almost everywhere and because this last function is measurable and its integral over the bounded set $\Omega\left(x_{0}\right)$ is finite by hypothesis, the Lebesgue Dominated Convergence Theorem [3] implies that

$$
\begin{aligned}
\lim _{h \rightarrow 0} \frac{1}{h} \int_{0}^{2 \pi} \int_{0}^{r\left(\theta, x_{0}\right)}\left(\varphi\left(u(\theta, s), x_{0}+h\right)-\varphi\left(u(\theta, s), x_{0}\right)\right)\left\|\frac{\partial u}{\partial \theta} \times \frac{\partial u}{\partial s}\right\| \mathrm{d} s \mathrm{~d} \theta= \\
\int_{0}^{2 \pi} \int_{0}^{r\left(\theta, x_{0}\right)} \frac{\partial \varphi}{\partial x}\left(u(\theta, s), x_{0}\right)\left\|\frac{\partial u}{\partial \theta} \times \frac{\partial u}{\partial s}\right\| \mathrm{d} s \mathrm{~d} \theta=\int_{\Omega\left(x_{0}\right)} \frac{\partial \varphi}{\partial x}\left(q, x_{0}\right) \mathrm{d} q .
\end{aligned}
$$

On the other hand, using the continuity of $\varphi$, one can deduce that

$$
\begin{gathered}
\lim _{h \rightarrow 0} \frac{1}{h} \int_{0}^{2 \pi} \int_{r\left(\theta, x_{0}\right)}^{r\left(\theta, x_{0}+h\right)} \varphi\left(u(\theta, s), x_{0}+h\right)\left\|\frac{\partial u}{\partial \theta}(\theta, s) \times \frac{\partial u}{\partial s}(\theta, s)\right\| \mathrm{d} s \mathrm{~d} \theta \\
=\lim _{h \rightarrow 0} \frac{1}{h} \int_{0}^{2 \pi} \int_{x_{0}}^{x_{0}+h} \varphi\left(u(\theta, r(\theta, z)), x_{0}+h\right)\left\|\frac{\partial u}{\partial \theta}(\theta, r(\theta, z)) \times \frac{\partial u}{\partial s}(\theta, r(\theta, z))\right\| \frac{\partial r}{\partial x}(\theta, z) d z \mathrm{~d} \theta \\
\quad=\int_{0}^{2 \pi} \varphi\left(u\left(\theta, r\left(\theta, x_{0}\right)\right), x_{0}\right)\left\|\frac{\partial u}{\partial \theta}\left(\theta, r\left(\theta, x_{0}\right)\right) \times \frac{\partial u}{\partial s}\left(\theta, r\left(\theta, x_{0}\right)\right)\right\| \frac{\partial r}{\partial x_{0}}\left(\theta, x_{0}\right) \mathrm{d} \theta .
\end{gathered}
$$

Since $\gamma(\theta, x)=u(\theta, r(\theta, x))$ for all $\theta \in \mathbb{S}^{1}$ and $x \in \mathcal{I}_{x_{0}}$, one has

$$
\begin{aligned}
& \frac{\partial \gamma}{\partial \theta}\left(\theta, x_{0}\right)=\frac{\partial u}{\partial \theta}\left(\theta, r\left(\theta, x_{0}\right)\right)+\frac{\partial u}{\partial s}\left(\theta, r\left(\theta, x_{0}\right)\right) \frac{\partial r}{\partial \theta}\left(\theta, x_{0}\right), \\
& \frac{\partial \gamma}{\partial x}\left(\theta, x_{0}\right)=\frac{\partial u}{\partial s}\left(\theta, r\left(\theta, x_{0}\right)\right) \frac{\partial r}{\partial x}\left(\theta, x_{0}\right) .
\end{aligned}
$$

Let $\chi$ denote the angle formed by $\frac{\partial \gamma}{\partial \theta}\left(\theta, x_{0}\right)$ and $\frac{\partial u}{\partial s}\left(\theta, r\left(\theta, x_{0}\right)\right)$. Then (omitting the expression $(\theta, r(\theta, x))$ for brevity),

$$
\left\|\frac{\partial u}{\partial \theta} \times \frac{\partial u}{\partial s}\right\|=\left\|\left(\frac{\partial u}{\partial \theta}+\frac{\partial u}{\partial s} \frac{\partial r}{\partial \theta}\right) \times \frac{\partial u}{\partial s}\right\|=\left\|\frac{\mathrm{d} \gamma}{\mathrm{d} \theta}\right\|\left\|\frac{\partial u}{\partial s}\right\| \sin \chi=\left\|\frac{\partial \gamma}{\partial \theta}\right\| n^{t}(\gamma) \frac{\partial u}{\partial s}
$$




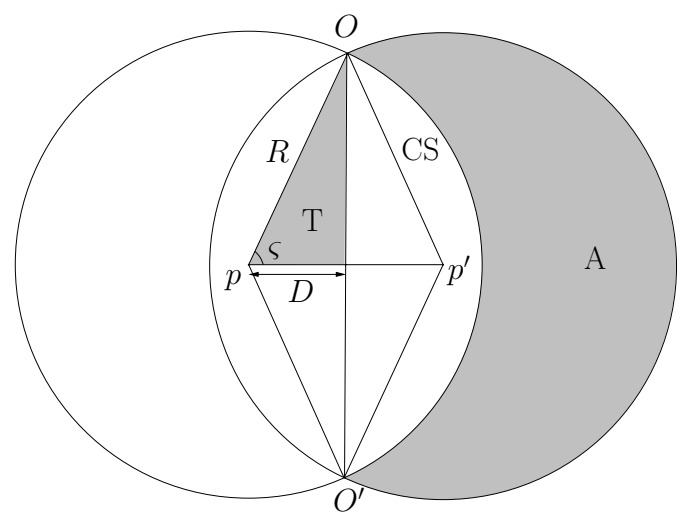

Figure 9. Areas of interest: $\mathrm{A}$ is the area of $\bar{B}\left(p^{\prime}, R\right) \cap \bar{B}(p, R)^{c}$, $\mathrm{T}$ is the area of the triangle $T\left(p, O, \frac{p^{\prime}+p}{2}\right)$, and CS is the area of the circular sector inside $\bar{B}(p, R)$ determined by $\left(p, O, O^{\prime}\right)$.

where in the last inequality we have used the fact that, since $\gamma_{x_{0}}$ is a parameterization of $\partial \Omega\left(x_{0}\right)$, then sin $\chi=$ $\cos \psi$, where $\psi$ is the angle formed by $n$, the outward normal to $\partial \Omega\left(x_{0}\right)$, and $\frac{\partial u}{\partial s}$. Therefore, we finally arrive at

$$
\begin{aligned}
& \int_{0}^{2 \pi} \varphi\left(\gamma(\theta), x_{0}\right) \| \frac{\partial u}{\partial \theta}\left(\theta, r\left(\theta, x_{0}\right)\right) \times \frac{\partial u}{\partial s}\left(\theta, r\left(\theta, x_{0}\right)\right) \| \frac{\partial r}{\partial x}\left(\theta, x_{0}\right) \mathrm{d} \theta \\
&=\int_{0}^{2 \pi} \varphi\left(\gamma(\theta), x_{0}\right)\left\|\frac{\partial \gamma}{\partial \theta}\left(\theta, x_{0}\right)\right\| n^{t}\left(\gamma\left(\theta, x_{0}\right)\right) \frac{\partial \gamma}{\partial x}\left(\theta, x_{0}\right) \mathrm{d} \theta=\int_{\partial \Omega\left(x_{0}\right)} \varphi\left(\gamma, x_{0}\right) n^{t}(\gamma) \frac{\partial \gamma}{\partial x} \mathrm{~d} \gamma
\end{aligned}
$$

Given the hypothesis of Proposition A.1, both terms in (22) and (23) have a continuous dependence on $x_{0} \in$ $(a, b)$, which concludes the proof.

Remark A.2. Note that the result in Proposition A.1 can be readily extended to any family of sets $\{\Omega(x) \mid x \in(a, b)\}$ that admits a suitable decomposition into piecewise smooth families consisting of strictly star-shaped sets. For instance, if $\left\{\Omega_{i}(x) \mid x \in(a, b)\right\}, i \in\{1,2\}$ are two piecewise smooth families with strictly star-shaped sets and we consider the family $\Omega(x)=\Omega_{1}(x) \backslash \Omega_{2}(x)$, for all $x \in(a, b)$, then the same result holds for the function

$$
x \in(a, b) \mapsto \int_{\Omega(x)} \varphi(q, x) \mathrm{d} q=\int_{\Omega_{1}(x)} \varphi(q, x) \mathrm{d} q-\int_{\Omega_{2}(x)} \varphi(q, x) \mathrm{d} q,
$$

by applying Proposition A.1 to each summand on the right-hand side of the equality.

\section{B. UPPER BOUND ON THE AREA OF THE INTERSECTION BETWEEN TWO BALLS}

Lemma B.1. For $R \in \overline{\mathbb{R}}_{+}$, let $p, p^{\prime} \in \mathbb{R}^{2}$ satisfy $\left\|p-p^{\prime}\right\| \leq R$. Then the area $A$ of $\bar{B}\left(p^{\prime}, R\right) \cap \bar{B}(p, R)^{c}$ satisfies $A \leq \frac{2 \sqrt{3}+3}{3} R\left\|p-p^{\prime}\right\|$.

Proof. The area A equals $\pi R^{2}-\mathrm{L}$, where $\mathrm{L}$ is the area of the non-trivial lune $\bar{B}(p, R) \cap \bar{B}\left(p^{\prime}, R\right)$ (see Fig. 9). Let $O, O^{\prime}$ be the two points in the intersection $\partial \bar{B}(p, R) \cap \partial \bar{B}\left(p^{\prime}, R\right)$. Note that the triangle with vertices $O$, $p$ and $p^{\prime}, T\left(O, p, p^{\prime}\right)$, and the triangle $T\left(O^{\prime}, p, p^{\prime}\right)$ are isosceles. This implies that the diagonals of the polygon $P\left(p, O, p^{\prime}, O^{\prime}\right)$ intersect at the middle point $\frac{p^{\prime}+p}{2}$. Let $\varsigma$ be the angle of $T\left(O, p, p^{\prime}\right)$ at $p$ and $D=\frac{\left\|p-p^{\prime}\right\|}{2}$. Then, the area of the lune $\mathrm{L}$ can be computed as $\mathrm{L}=2(\mathrm{CS}-2 \mathrm{~T})$, where CS is the area of a circular sector with angle $2 \varsigma$ and $\mathrm{T}$ is the area of the triangle $T\left(p, O, \frac{p^{\prime}+p}{2}\right)$. Since $\mathrm{CS}=\varsigma R^{2}$ and $\mathrm{T}=\frac{1}{2} D \sqrt{R^{2}-D^{2}}$, we have that 
$\mathrm{A}=R^{2}(\pi-2 \varsigma)+2 D \sqrt{R^{2}-D^{2}}$. Now, using that $0 \leq D \leq R$, we deduce that

$$
\mathrm{A} \leq R^{2}(\pi-2 \varsigma)+2 R D .
$$

In order to finally bound the first term of the right-hand side of the inequality with a quantity proportional to $D$, we use that $\varsigma=\arccos \left(\frac{D}{R}\right)$. Consider now the function $g(x)=\pi-2 \arccos x-K x$. It is easy to see that for $K>\frac{4}{\sqrt{3}}$, one has $g^{\prime}(x) \leq 0$ and $g(x) \leq 0$ when $0 \leq x \leq \frac{1}{2}$. In particular this implies that, for $0 \leq \frac{D}{R} \leq \frac{1}{2}$, we have that $\pi-2 \arccos \left(\frac{D}{R}\right) \leq K \frac{D}{R}$. In other words, the former inequality is valid for $\left\|p-p^{\prime}\right\| \leq R$. This concludes the proof.

\section{Discrete-time LaSalle Invariance Principle}

The following result is an extension of two classical results: on the one hand, it extends the discrete-time version of LaSalle Invariance Principle [19] to algorithms defined via set-valued maps. On the other hand, it considers a more general notion of Lyapunov function ( $c f$. Sect. 3.2) than in the usual statement of the Global Convergence Theorem [21]. After the submission of this manuscript, it has been brought to our attention that a related result is presented in [30].

Theorem C.1 (discrete-time LaSalle Invariance Principle). Let $T$ be a closed algorithm on $W \subset \mathbb{R}^{N}$ and let $U$ be a Lyapunov function for $T$ on $W$. Let $x_{0} \in W$ and assume the sequence $\left\{x_{n} \mid n \in \mathbb{N} \cup\{0\}\right\}$ defined via $x_{n+1} \in T\left(x_{n}\right)$ is in $W$ and bounded. Then there exists $c \in \mathbb{R}$ such that

$$
x_{n} \longrightarrow M \cap U^{-1}(c),
$$

where $M$ is the largest weakly positively invariant set contained in

$$
\left\{x \in \mathbb{R}^{N} \mid \exists y \in T(x) \text { such that } U(y)=U(x)\right\} \cap \bar{W} .
$$

Proof. Let $\Omega\left(x_{n}\right) \subset \bar{W}$ denote the $\omega$-limit set of the sequence $\left\{x_{n} \mid n \in \mathbb{N} \cup\{0\}\right\}$. First, let us prove that $\Omega\left(x_{n}\right)$ is weakly positively invariant. Let $x \in \Omega\left(x_{n}\right)$. Then there exists a subsequence $\left\{x_{n_{m}} \mid m \in \mathbb{N} \cup\{0\}\right\}$ of $\left\{x_{n} \mid n \in \mathbb{N} \cup\{0\}\right\}$ such that $x_{n_{m}} \rightarrow x$. Consider the sequence $\left\{x_{n_{m}+1} \mid m \in \mathbb{N} \cup\{0\}\right\}$. Since this sequence is bounded, it has a convergent subsequence. For ease of notation, we use the same notation to refer to it, i.e., there exits $y$ such that $x_{n_{m}+1} \rightarrow y$. By definition, $y \in \Omega\left(x_{n}\right)$. Moreover, using the fact that $T$ is closed, we deduce that $y \in T(x)$. Therefore $\Omega\left(x_{n}\right)$ is weakly positively invariant.

Now, consider the sequence $\left\{U\left(x_{n}\right) \mid n \in \mathbb{N} \cup\{0\}\right\}$. Since $\left\{x_{n} \mid n \in \mathbb{N} \cup\{0\}\right\}$ is bounded and $U$ is a Lyapunov function for $T$ on $W$, this sequence is decreasing and bounded from below, and therefore convergent. Let $c \in \mathbb{R}$ such that $U\left(x_{n}\right) \rightarrow c$. Let us see that the value of $U$ on $\Omega\left(x_{n}\right)$ is constant and equal to $c$. Take any $x \in \Omega\left(x_{n}\right)$. Accordingly, there exists a subsequence $\left\{x_{n_{m}} \mid m \in \mathbb{N} \cup\{0\}\right\}$ such that $x_{n_{m}} \rightarrow x$. Since $U$ is continuous, $U\left(x_{n_{m}}\right) \rightarrow U(x)$. From $U\left(x_{n}\right) \rightarrow c$, we conclude that $U(x)=c$.

Finally, the fact that $\Omega\left(x_{n}\right)$ is weakly positively invariant and $U$ is constant on $\Omega\left(x_{n}\right)$, implies that

$$
\Omega\left(x_{n}\right) \subset\left\{x \in \mathbb{R}^{N} \mid \exists y \in T(x) \text { such that } U(y)=U(x)\right\} .
$$

Therefore, we conclude that $x_{n} \rightarrow M \cap U^{-1}(c)$, where $M$ is the largest weakly positively invariant set contained in $\left\{x \in \mathbb{R}^{N} \mid \exists y \in T(x)\right.$ such that $\left.U(y)=U(x)\right\} \cap \bar{W}$.

\section{REFERENCES}

[1] R.C. Arkin, Behavior-Based Robotics. Cambridge, Cambridge University Press (1998).

[2] Y. Asami, A note on the derivation of the first and second derivative of objective functions in geographical optimization problems. J. Faculty Engineering, The University of Tokio (B) XLI (1991) 1-13. 
[3] R.G. Bartle, The Elements of Integration and Lebesgue Measure, 1st edn. Wiley-Interscience (1995).

[4] M. de Berg, M. van Kreveld and M. Overmars, Computational Geometry: Algorithms and Applications. New York, SpringerVerlag (1997).

[5] S. Boyd and L. Vandenberghe, Convex Optimization. Cambridge, Cambridge University Press (2004).

[6] A.J. Chorin and J.E. Marsden, A Mathematical Introduction to Fluid Mechanics. 3rd edn., Ser. Texts in Applied Mathematics. New York, Springer-Verlag 4 (1994).

[7] J. Cortés and F. Bullo, Coordination and geometric optimization via distributed dynamical systems. SIAM J. Control Optim. (June 2004), to appear.

[8] J. Cortés, S. Martínez, T. Karatas and F. Bullo, Coverage control for mobile sensing networks. IEEE Trans. Robotics Automat. 20 (2004) 243-255.

[9] R. Diestel, Graph Theory. 2nd edn., Ser. Graduate Texts in Mathematics. New York, Springer-Verlag 173 (2000).

[10] Z. Drezner and H.W. Hamacher, Eds., Facility Location: Applications and Theory. New York, Springer-Verlag (2001).

[11] Q. Du, V. Faber and M. Gunzburger, Centroidal Voronoi tessellations: applications and algorithms. SIAM Rev. 41 (1999) $637-676$.

[12] H. Edelsbrunner and N.R. Shah, Triangulating topological spaces. Internat. J. Comput. Geom. Appl. 7 (1997) $365-378$.

[13] J. Gao, L.J. Guibas, J. Hershberger, L. Zhang and A. Zhu, Geometric spanner for routing in mobile networks, in ACM International Symposium on Mobile Ad-Hoc Networking \& Computing (MobiHoc). Long Beach, CA (Oct. 2001) 45-55.

[14] R.M. Gray and D.L. Neuhoff, Quantization. IEEE Trans. Inform. Theory 44 (1998) 2325-2383. Commemorative Issue 1948-1998.

[15] U. Helmke and J. Moore, Optimization and Dynamical Systems. New York, Springer-Verlag (1994).

[16] A. Jadbabaie, J. Lin and A.S. Morse, Coordination of groups of mobile autonomous agents using nearest neighbor rules. IEEE Trans. Automat. Control 48 (2003) 988-1001.

[17] J.W. Jaromczyk and G.T. Toussaint, Relative neighborhood graphs and their relatives. Proc. of the IEEE 80 (1992) $1502-1517$.

[18] H.K. Khalil, Nonlinear Systems. Englewood Cliffs, Prentice Hall (1995).

[19] J.P. LaSalle, The Stability and Control of Discrete Processes. Ser. Applied Mathematical Sciences. New York, Springer-Verlag 62 (1986).

[20] X.-Y. Li, Algorithmic, geometric and graphs issues in wireless networks. Wireless Communications and Mobile Computing 3 (2003) 119-140.

[21] D.G. Luenberger, Linear and Nonlinear Programming. Reading, Addison-Wesley (1984).

[22] J. Marshall, M. Broucke and B. Francis, Formations of vehicles in cyclic pursuit. IEEE Trans. Automat. Control 49 (2004) $1963-1974$

[23] A. Okabe, B. Boots, K. Sugihara and S.N. Chiu, Spatial Tessellations: Concepts and Applications of Voronoi Diagrams. 2nd edn., Ser. Wiley Series in Probability and Statistics. New York, John Wiley \& Sons (2000).

[24] A. Okabe and A. Suzuki, Locational optimization problems solved through Voronoi diagrams. European J. Oper. Res. 98 (1997) 445-56.

[25] A. Okubo, Dynamical aspects of animal grouping: swarms, schools, flocks and herds. Adv. Biophysics 22 (1986) 1-94.

[26] R. Olfati-Saber and R.M. Murray, Consensus problems in networks of agents with switching topology and time-delays. IEEE Trans. Automat. Control 49 (2004) 1520-1533.

[27] K.M. Passino, Biomimicry for Optimization, Control, and Automation. New York, Springer-Verlag (2004).

[28] C.W. Reynolds, Flocks, herds, and schools: A distributed behavioral model. Computer Graphics 21 (1987) 25-34.

[29] K. Rose, Deterministic annealing for clustering, compression, classification, regression, and related optimization problems. Proc. of the IEEE 80 (1998) 2210-2239.

[30] A.R. Teel, Nonlinear systems: discrete-time stability analysis. Lecture Notes, University of California at Santa Barbara (2004). 